Ann. Abeille, I966, 9 (2), 83-II4.

\title{
LE COMPORTEMENT PRÉDATEUR DU CRAPAUD COMMUN (BUFO BUFO) ENVERS LES ABEILLES
}

\author{
J. LESCURI: \\ Séminaire-Collège, Fort-de-France (Martinique)
}

SOMMAIRE

Le Crapaud commun a la réputation de venir happer les $A$ beilles au bas des ruches? Prend-il l'habitude d'une telle proie malgré les piqûres? Il semble y avoir contradiction apparente entre les travaux de CotT (I936) et les constatations de plusieurs apiculteurs.

En fait, le soir, lorsque les abeilles sont moins agressives, de gros crapauds peuvent s'adapter à une proie aussi dangereuse.

Bufo bufo, d'ailleurs, ne réussit pas à apprendre à refuser totalement les abeilles. Celles-ci sont des proies ambivalentes, elles constituent tout aussi bien un signal de récompense (bonne au goût) qu'un signal de punition (piqûre). I.e Crapaud oublie vite la punition et recommence périodiquement à avaler des abeilles.

\section{INTRODUCTION}

IE CRAPAUD EST-IL UN ENNEMI DES ABEILILES?

\section{a) Aperçu historique}

S'il est une proie que les Crapauds ont la réputation de manger facilement, ce sont bien les Abeilles. BoulANGER (IgIo), spécialiste incontesté des Batraciens, écrivait au début du siècle que " $B u f o$ bufo était friand d'Abeilles et se tenait volontiers dans le voisinage des ruches pour happer ces Insectes au passage ". C'était 1'opinion qui prévalait de son temps et cette réputation débordait les milieux del'Apiculture. A la fin du XIX ${ }^{e}$ siècle, ce sujet fut longuement débattu entre naturalistes. Je crois que la question fut abordée pour la première fois avec clarté par Alphonse de LA FONTAINE (I870); ses observations furent malheureusement trop ignorées par la suite. Les beaux soirs d'été, il avait vu des crapauds escalader la planchette d'envol et se mettre en embuscade devant l'ouverture d'une ruche, puis happer les abeilles. Les dégâts auraient été importants si l'apiculteur n'avait pas tué, en l'espace d'un mois, plus de 200 crapauds près de son rucher de 60 essaims. 
Fatio (I872) cita La Fontaine; Collin de Plancy (I874) et Héron-Royer (I878) connaissaient l'article de FATIO, mais ne tinrent pas compte de l'observation de La Fontaine. Défenseur acharné de l'utilité agricole de Bufo bujo, Héron-Royer critiqua le catalogue de GIRARD (I878), qui jugeait les Anoures utiles, mais avait classé $B$ ufo parmi les nuisibles aux Abeilles.

Après BOULENGER, 1'idée du Crapaud " friand d'Abeilles ", est encore affirmée (Rostand, I933; de WiTte, I942). Cela pouvait être vraisemblable, puisque Mme Phisalix (I922) n'avait constaté chez lui aucun symptôme d'envenimement dû à des piqûres d'Abeilles. RosTAND observa la même absence de réactions inflammatoires aux piqûres de Guêpes.

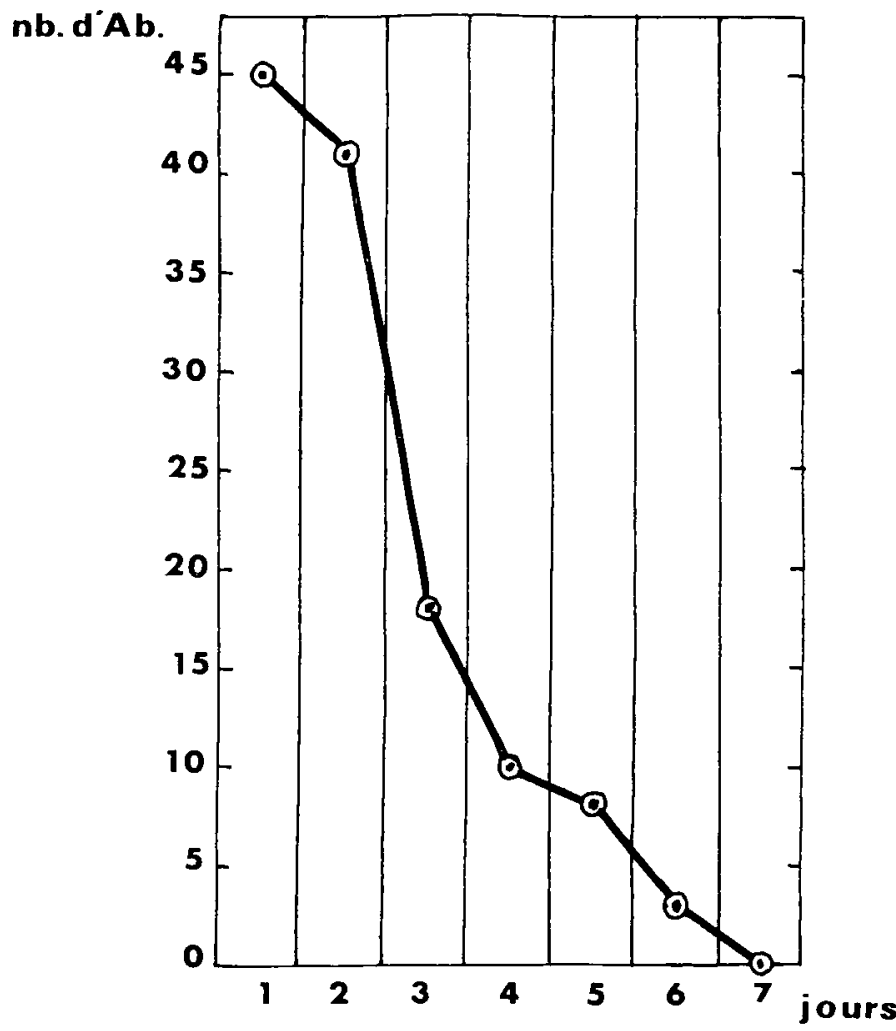

FIG. I. - (СотT). Nombre d'abeilles avalées par les 34 crapauds pendant 7 jours conséculifs

Les piqûres d'abeilles seraient-elles inefficaces contre un prédateur comme le Crapaud? CotT (1936) n'est pas de cet avis et, apparemment, les résultats de ses travaux contredisent une telle opinion.

L'expérience du zoologiste anglais consistait à placer des crapauds affamés sur la planche d'envol d'une ruche devant ses orifices de sortie, puis à observer leurs réactions. Pour écarter les facteurs ; hasard, effet climatique et variabilité de comportement des essaims, il avait pris la précaution de travailler sur une grande échelle ( 34 Crapauds, 7I4 essais pendant plus de $40 \mathrm{~h}$ ) et à des époques différentes. Il avait choisi 7 ruches moyennement actives et faisait jeûner les Bufo durant une semaine 
avant l'expérience. Comme l'indique ses figures I et 2, plus aucun Crapaud ne mangea d'Abeilles le $7^{\mathrm{e}}$ jour mais beaucoup s'élancèrent sur les Vers de farine après l'expérience $(7 \mathrm{a})$.

CoTT concluait : " même dans des conditions de famine et en dépit d'une faim croissante, ces crapauds apprennent, au bout de quelques essais seulement, à éviter les abeilles bien que d'autres Insectes (Vers de farine) soient acceptés avec empressement. "

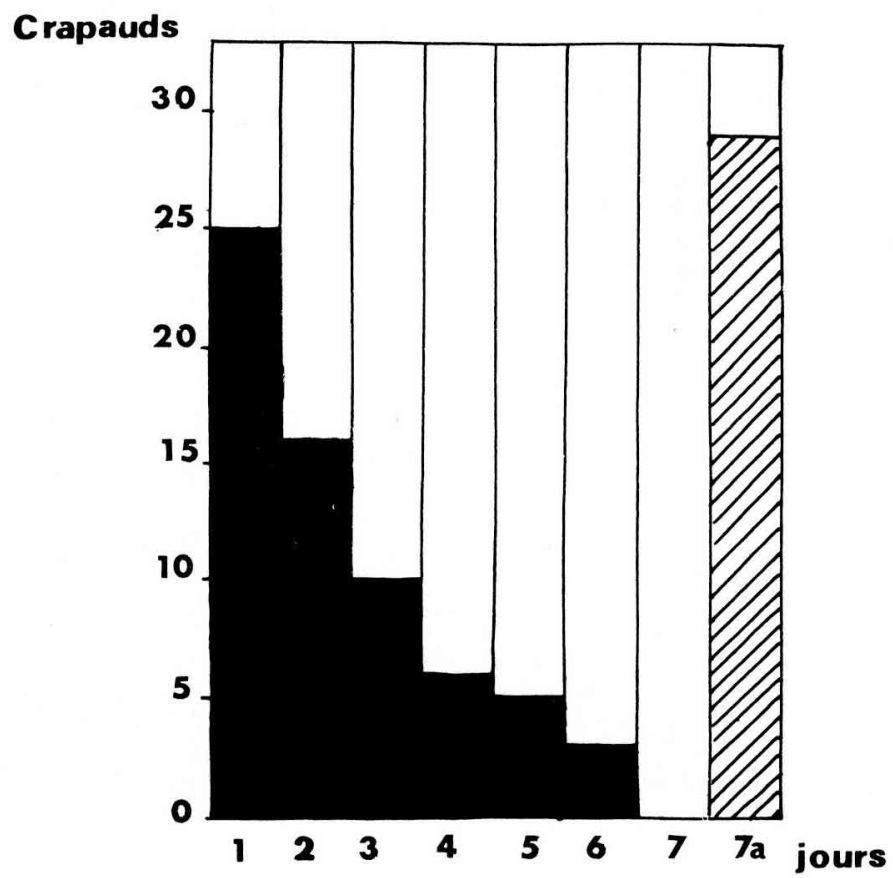

FIG. 2. - (СОтT). Nombre de Crapauds avalant des Abeilles chaque jour el nombre de ceux qui avalèrent des vers de farine à la fin de l'expérience ( 7 a)

\section{b) Les Crapauds et l'Apiculture}

Comme l'a relevé Chauvin (r956) les conclusions de Cotr ne concordent pas avec les faits rapportés encore actuellement par des apiculteurs, particulièrement par ceux d'Afrique du Nord ou d'Amérique du Sud. Mais les Crapauds, auxquels ceux-ci font allusion, ne sont pas des Bufo bufo; je pense qu'il s'agit pour l'Afrique du Nord, de Bufo bufo spinosus et même de grands Bufo viridis et pour l'Amérique du Sud de Bufo marinus. Ces espèces sont plus grandes que celles de notre pays. Un ancien apiculteur de la Martinique, le Père Delawarde, m'a décrit ses observations de capture d'abeilles par Bufo marinus, ces gros crapauds causent de réels dégâts quand ils réussissent à s'approcher des orifices de sortie d'une ruche. Chapman Grant (I948) qui conteste aussi l'expérience de Cotrt, cite une lettre d'un apiculteur des Bermudes qui avait trouvé 30 à 50 Bufo marinus opérant dans un rucher. En ouvrant les tubes digestifs d'une douzaine d'entre eux, il compta une moyenne de 50 à 60 abeilles pat estomac; le maximum fut de II3 chez un grand spécimen. 
Il faut mentionner également l'observation très précise d'ECKERT en I934: durant des nuits de lune, ce zoologiste a capturé I 6 Crapauds de Californie (8 à I I cm) aux environs de I2 ruches. Les déjections de Crapauds trouvées autour du rucher étaient pleines de têtes d'Abeilles, 5I4 têtes d'Abeilles furent comptées dans les estomacs de 22 animaux, le maximum étant de 38 et le minimum de 13.

En France, dans les traités de vulgarisation apicole, les affirmations ne sont pas aussi nettes qu'on pourrait le croire en ce qui concerne le Crapaud "ennemi " des Abeilles. Sur 35 livres recensés, les plus connus dans notre pays, I3 seulement en font mention et accompagnent souvent leurs déclarations de beaucoup de réserve. Généralement, il n'est pas considéré comme un ennemi bien dangereux. Seul des 35 auteurs, AlPHANDÉRy (r946), dans l'Encyclopédie apicole, relate un fait précis signalé par FLAMMARION. La plupart du temps, les ouvrages indiquent le remède le plus commode et le plus efficace contre l'intrusion du Crapaud : surélever la ruche en la haussant sur pieds, le Crapaud ne peut plus atteindre la planche d'envol.

Les ruches étant souvent surélevées à l'heure actuelle, je me suis demandé $\mathrm{s}^{\mathrm{i}}$ réellement la chasse aux Abeilles était encore possible pour le Crapaud commun, Bufo bufo. J'ai effectué un sondage par l'intermédiaire de la Gazette apicole (I5 ooo abonnés), demandant aux apiculteurs s'ils avaient vraiment vu des crapauds happer des abeilles. Plusieurs réponses me parvinrent ou arrivèrent à la revue, de diverses régions de France : Bretagne, Lorraine, Drôme, Provence. Elles révèlent parfois d'astucieuses adaptations individuelles, de la part de ces Vertébrés inférieurs. Ainsi, M. GrémrLLoN me signale un cas curieux, observé en septembre I96I. Dans sa propriété d'Orange existe un assez grand bassin avec, au centre, une rocaille d'où jaillit un jet d'eau. Vers $6 \mathrm{~h}$ du matin, les abeilles, très nombreuses, viennent s'y abreuver ; un jour, il remarqua à $7 \mathrm{~h}$ un gros crapaud qui traversa le bassin à la nage, monta sur la rocaille, puis happa les abeilles sans difficulté. Encore plus étonnant est le fait contrôlé par le Dr Moreaux avec Cuḱnot et relaté dans la Gazette apicole de février r 962.

"Nous avions remarqué un jour, un gros crapaud qui se tenait à l'affût sous la planche d'envol d'une ruche isolée, happant au passage les butineuses qui, alourdies au retour d'un voyage de récolte, se laissaient choir dans 1'herbe. Quelques jours plus tard ce crapaud avait construit à la verticale de la planche d'envol une cavité en entonnoir au fond de laquelle il se tenait, se nourrissant des abeilles qui chutaient dans l'orifice de sa demeure.

Ayant déterré ce crapaud, nous l'avons rejeté à une dizaine de mètres de la ruche. Or, deux jours plus tard, il avait reconstruit et réintégré son logis.

"Déterré à nouveau, nous l'avons bagué à une patte d'un cordonnet de soie rouge et l'avons rejeté cette fois au-dessus d'une haie voisine. Mais une semaine plus tard, nous retrouvions ce même crapaud dans son logis à notveau reconstruit.

"Intrigués de sa ténacité, nous l'avons alors porté à 200 mètres environ de la ruche, mais quelle n'a pas été ma surprise de retrouver l'année suivante notre même crapaud, dûment bagué, au fond dı même entonnoir où à nouveaut, il se délectait d'abeilles. ")

" Je décidai alors de le sacrifier et de le disséquer et trouvai dans son estomac les débris de plus de quarante abeilles. ")

On peut donc affirmer que des crapauds viennent encore au bas des ruches happer des abeilles. L'observation de LA FontTaINE (I870) n’était pas un cas isolé. Les Cra- 
pauds peuvent guetter les abeilles fatiguées, traînant autour des ruches, ou s'approcher de la planche d'envol, si celle-ci n'est pas suffisamment surélevée comme je l'ai constaté en Provence. Mais je ne pense pas, malgré les dires de certains apiculteurs, que le Crapaud happe l'Abeille au vol. Personnellement, je ne l'ai observé que quelques fois avec un crapaud borgne. Au contraire, le vol et l'atterrissage très rapide des abeilles provoquent des réactions de fuite ou d'immobilité du Crapaud. Quant à leur pouvoir de fascination sur les Abeilles que laissent entendre des récits beaucoup trop anthropomorphiques (M. RichardeAu, Gazette apicole, janvier I962), il s'agit bien d'une légende : les Abeilles, guettées attentivement par le Crapaud immobile, ne modifient pas pour autant leur comportement.

\section{c) Situation actuelle de la question}

$\mathrm{Si}$ des crapauds viennent avaler les abeilles près des ruches, peut-on affirmer à cause des expériences de CoTT, que ces Batraciens peuvent s'habituer à une proie aussi " dangereuse " et trouver dans un rucher une source de nourriture abondante et d'accès facile? Je pense que le problème n'est toujours pas éclairci malgré les quelques faits signalés par des apiculteurs. Dotrresss (rg63) résume bien la situation actuelle lorsqu'il écrit : "On prétend qu'il (1e Crapaud) pille les ruches ; pourtant l'expérience a montré qu'il apprend à éviter les Abeilles parce qu'elles le piquent quand elles sont happées. Peut-être certains individus s'accoutument-ils à cet inconvénient? ” La réponse à la question de DotTrens ne peut être apportée que par une vérification expérimentale comme j'ai tenté de le faire.

Il s'avère aussi que cette expérience d'adaptation du Crapaud à 1'Abeille fournit en même temps un très bon exemple d'étude de comportement acquis envers une proie ambivalente. C'est un cas d'apprentissage " positif " $\left.{ }^{1}\right)$ où se mêlent la récompense (1'Abeille, bonne au goût) et la punition (piqûre). La récompense l'emporte t-elle sur la punition? La punition est-elle assez forte pour écarter le Crapaud de la proie? Ce type d'expérience n'a jamais été réalisé jusqu'ici avec un Batracien.

\section{II. - MÉTHODE ET MATÉRIEI. \\ I. - Constatations prúliminaires}

Le dessein de CớT était d'abord de prouver l'efficacité des adaptations protectrices des Abeilles contre les attaques prédatrices de Bufo bufo, puis de jeter quelques lumières sur le comportement alimentaire, le pouvoir d'association et la mémoire de ces crapauds. Mon but est tout à fait différent, il est à l'opposé de celui de Cotr : les Crapauds s'adaptent-ils à la capture d'une proie aussi dangereuse ? Ceci m'a amené à modifier diverses conditions d'expériences qui, maintenues, auraient entraîné pour les Crapauds des résultats d'apprentissage trop négatifs.

(1) J'appelle apprentissage "positif " celui qui est lié à l'acquisition d'une récompense ; il s'établit à partir d'un stimulus " positif" (LE NY, I 96I) qui produit un effet excitatif. Le dressage "négatif " est lié à un stimulus " négatif " qui produit un effet inhibitif, il aboutit, après apprentissage, à une réaction d'évitement. 


\section{a) Importance de l'heure}

Au cours de ma première série d'expériences (Série $A$, I er essai) où je reproduisais le cadre expérimental de Cơ⿰氵, j'ai nettement perçu que le vol fréquent des abeilles " gênait " les crapauds ; c'était même la principale cause de leur fuite. Lorsque je parle de "gêne » je veux parler du fait que lorsque le Crapaud est sur la planche d'envol les abeilles se posent sur lui à leur retour et grimpent sur son dos, l'obligeant à rester immobile et à fermer les yeux. Finalement, il m'a semblé que ce n'étaient pas les piqûres ni la déglutition de la proie, mais ce bourdonnement et ce va-et-vient continuel qui provoquaient la fuite.

Lors du deuxième essai de la série $A$, j'ai supprimé ce facteur en travaillant à la tombée de la nuit quand les vols sont quasiment ou même complètement terminés. Le Crapaud peut alors se mettre à l'affût tout près des orifices de sortie. Comme Bufo bufo est un animal crépusculaire, ces heures tardives sont plus habituelles pour lui. Dans la nature, c'est le soir que les Crapauds s'approchent des ruches. J'ajouterai aussi que $B$ fo a presque toujours une tendance instinctive à gagner l'ombre (BuyTENDIJK, I928) : il fuira rapidement d'une planche d'envol ensoleillée, même s'il a 1'habitude de manger pendant le jour. Le même spécimen restera plus calme le soir.

CoT'T ne précise pas l'heure de ses expériences, mais elles devaient se dérouler durant la journée, car il parle des vols assez fréquents d'abeilles, à tel point qu'il était obligé d'exclure les ruches trop actives.

\section{b) Importance de la manipulation}

Très vite, j'ai noté un facteur capable de perturber facilement le déroulement normal d'une expérience et qui malheureusement ne pouvait pas être éliminé totalement : la manipulation de l'animal par l'expérimentateur. Ces Batraciens, aux réactions individuelles très diverses, sont toujours très craintifs. Sous l'effet de ces manipulations ou d'un geste brusque, un réflexe de fuite peut se déclencher et inhiber tout autre comportement pendant un temps plus ou moins long, même après une période convenable d'apprivoisement.

On le constate aisément, lorsque le Crapaud, posé la nuit sur la plate-forme d'une ruche très calme, cherche à s'enfuir aussitôt, alors qu'il n'a jamais guetté les abeilles et qu'il n'a été ni attaqué ni piqué. Durant la journée et même au crépuscule, le bourdonnement et le vol des abeilles provoquant les mêmes réactions de fuite, il est plus difficile d'en déceler le véritable motif. Des crapauds qui, durant toute l'expérience, n'ont pas mangé une seule abeille, n'ont pas forcément appris à les éviter, puisqu'ils ne les ont pas goûtées et n'ont même pas été piqués; ils éritent peut-être systématiquement la ruche à cause du tourbillonnement, du bourdonnement, des vols incessants d'abeilles, mais la manipulation a dû contribuer à provoquer cette fuite automatique.

CoTT signale que ses crapauds s'étaient accoutumés à la société humaine et à être saisis avec les mains. Je pense qu'il n'a pas assez souligné cette action perturbatrice, car les réactions de certains spécimens, même habitués, sont très changeantes en ce domaine.

\section{c) Importance des réactions du Crapaud aux piqûres d'Abeilles}

Dès les premières observations, je me suis vite heurté à une difficulté : savoir quand un crapaud a été piqué par une abeille qu'il avale. Un seul cas est facilement 
observable, mais il est peu fréquent, la piqûre sur la langue. Ia réaction du Crapaud est instantanée, il rouvre aussitôt la bouche, bave un peu et laisse pendre la langue. Très souvent, $j$ 'y voyais le dard, mais je n'ai pu y constater aucune enflure. Des crapauds, comme $D_{1}$, pouvaient de nouveau happer des abeilles, Io minutes après avoir été piqués sur la langue ; mais généralement les autres s'éloignaient aussitôt de la ruche. Lorsque je ne voyais pas le dard sur l'extrémité de la langue pendant hors de la boutche, je le retrouvais toujours fixé sur l'autre partie de la langue, en ouvrant la gueule du crapat1d. Il arrive que la réaction décrite plus haut ne se manifeste pas si l'Abeille a piqué de gros crapauds comme $\mathrm{D}_{1}$ à la base de la langue.

En dehors de la piqûre sur la langue, ces "piqûres internes" comme les nomme COTT, sont signalées à l'expérimentateur par différents comportements, notamment un mouvement typique de crispation du cou et de la tête, durant une fraction de seconde, les yeux enfoncés dans leurs orbites. Souvent des clignements d'yeux. des gonflements et des dégonflements rapides des poumons suivent cette réaction "douloureuse ». Parfois le crapaud quitte aussitôt la planche d'envol et si des abeilles s'approchent de lui, il recule avec une brusquerie qui contraste singulièrement avec son calme antérieur. Je n'ai jamais constaté ces crispations de la tête durant la manducation d'une proie normale. Je les ai retrouvées lorsqu'un crapaud moyen avale un gros Hanneton (les pattes du Coléoptère doivent griffer les parois de la cavité buccale) mais la crispation et le spasme étaient bien moins accentués et plus brefs qu'avec les Abeilles.

Vérification expérimentale. - Le moyen de vérifier si une piqûre correspond à ces signes de " malaise " est d'ouvrir la gueule du crapaud pour voir si le dard est planté dans l'épithélium buccal.

Je ne pouvais pas entreprendre de telles observations durant mes expériences, car j'aurais perturbé le comportement du Crapaud pour plusieurs jours ; C'est pourquoi j'ai réalisé des séances spéciales avec I2 Bufo bufo. Par contre, le cas oì les Crapauds seraient piqués dans l'estomac est invérifiable in vivo Mais est-il possible? Je le pense, car des abeilles toutes froissées que j'avais fait dégurgiter, avaient encore un dard sortant de son fourreau et réagissant à une excitation tactile (frottements). BRAGG (I948) a constaté cette possibilité dans un cas analogue, puisqu'il a trouvé, dans des dissections, des parois d'estomacs de Crapauds américains hérissées de dards de Fourmis. Il est donc vraisemblable que des dards puissent encore piquer la paroi de l'estomac et s'y fixer. Ceci expliquerait peut-être certains comportements où le Crapaud n'a aucune réaction immédiate lors de la préhension et de la déglutition, mais manifeste, après un petit moment, plusieurs gonflements et dégonflements successifs du ventre.

Liaisons piqûre-malaise: expériences. Ces expériences effectuées avec I2 Bufo bufo se déroulèrent exactement dans le même cadre que les autres. 8 crapauds furent retirés et examinés après leur premier signe de " malaise " ou leur fuite ( $\mathrm{n}^{0}$ III à X). 2 autres (I et II) furent retirés alors qu'ils n'avaient manifesté encore aucun de ces comportements tandis que les deux derniers (XI et XII) avaient réagi plusieurs fois.

Commentaire. - Les dards que je retrouvais étaient, la plupart du temps, fixés au fond de la gorge; quelques-uns s'étaient accrochés aux filaments visqueux de la salive et offraient peu de résistance quand ils étaient enlevés. Le nombre de dards retrouvés n'égale pas le nombre d'abeilles avalées et ne correspond pas toujours au nombre de réactions de "malaise " ou de fuite. 
Si le nombre de réactions est supérieur ( $n^{\circ}$ III et VII), ce serait soit parce que le dard a mal piqué et a été finalement avalé (?), soit plutôt parce que l'abeille a piqué l'œesophage ou l'estomac. L,e « malaise » est généralement plus accentué lorsque le dard est fixé dans la vonte du palais, mais il n'y a jamais d'enflure. Le comportement de fuite n'est pas forcément la conséquence d'une piqûre, car il peut être provoqué par d'autres facteurs ; mais il en est certainement le signe lorsque au cours d'une expérience portant sur la capture d'une seule abeille, il se présente immédiatement après la préhension de l'Insecte.

TABLEAU I

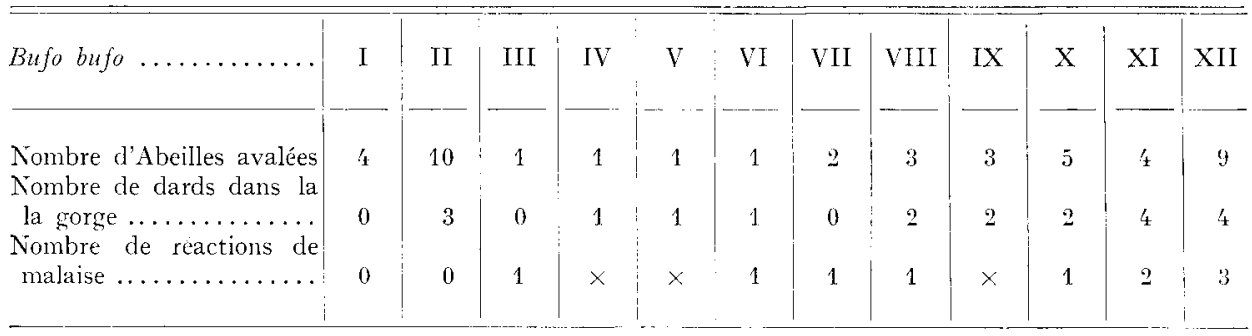

$x$ indique une fuite immédiate après avoir happé une Abəille sans réaction " douloureuse ".

Si le nombre de dards est supérieur aux réactions, c'est donc que des piqûres auraient été peu douloureuses. I, Crapaud serait-il peu sensible à l'action du venin. d'Apis mellifica?

M. Phisalix (I922) parle d'une véritable immunité naturelle du Crapaud à ce venin. En quelques expériences j'ai voulu examiner l'ampleur de cette immunité naturelle; j’ai essayé de tester le degré de résistance du Crapaud en lui inoculant différentes doses de venin d'Abeille.

\section{Expériences}

Pour me procurer le venin d'Abeille, je laissais tremper les appareils venimeux entiers dans de l'eau physiologique ou de l'eau distillée. On extrait les appareils venimeux en saississant et en tirant le dard de l'Abeille hors de son fourreau. Toute la glande venimeuse vient avec l'appareil inoculateur.

Parfois, la glande est collée à la paroi de l'intestin, mais les deux sont facilement séparables. L'appareil venimeux, plongé dans l'eau, vide son réservoir à venin. On aperçoit les muscles de l'appareil venimeux effectuer les mêmes mouvements que lorsque le dard est accroché à un épiderme. Les glandes étaient légèrement broyées pour faciliter la dissolution.

La solution était inoculée aussitôt ou utilisée dans les jours suivants, la conservation à $-40^{\circ}$ n'apportant pas de modification au venin. La grande variabilité de la quantité et de la toxicité du venin de chaque Abeille empêche d'évaluer avec précision le contenu type d'une glande à venin. J'ai donc calculé la concentration de la solution obtenue en comptant le nombre de glandes par $\mathrm{cm}^{3}$.

\section{Première série d'expériences}

Résultats.

I. Solution de 10 glandes par $\mathrm{cm}^{3}$. durable.

Elle est injectée à 6 crapauds d'environ $20 \mathrm{~g}$ dans le cou, les flancs ou la langue. Aucun effet 
2. Solution de 40 glandes dans $0,8 \mathrm{~cm}^{3}$.

Cette solution est injectée dans la cuisse et la patte antérieure droite d'un crapaud ơ de $23 \mathrm{~g}$.

Au moment de l'injection, l'animal crie et se débat. Presque aussitôt se produisent des clignements d'yeux et une sécrétion des glandes sudoripares pustuleuses de la patte. La patte antérieure est paralysée. Mort survenue après 20 heures.

3. Solution de 30 glandes dans $0,6 \mathrm{~cm}^{3}$.

Cet te solution est inoculée dans l' "avant-bras " d'un crapaud ơ de $24 \mathrm{~g}$.

La patte antérieure droite s'est enflée instantanément, elle est ramenée sous le corps du crapaud et ne sert plus à la marche. Les doigts sont recroquevillés, la main violacée saigne un peu. Trois heures après, la main toujours très enflée saigne encore. Après 5 heures, le crapaud ne réagit plus. Après ro heures, il meurt.

Lot témoin : cinq Bufo bufo ơ de 20 à $25 \mathrm{~g}$ ont été piqués avec les mêmes aiguilles. On leur inocula des doses identiques d'eau physiologique, solvant du venin d'Abeille, dans les flancs, la langue ou les pattes de devant. Aucune enflure ne fut constatée dans les flancs. Les pattes étaient enflées, mais les crapauds s'en servaient pour la marche comme avant l'opération.

\section{Deuxième série d'expériences}

J.e venin est dissous dans de l'eau physiologique et inoculé dans les muscles de la patte de devant.

Résultats

I. 30 glandes à venin dissoutes dans $0,8 \mathrm{~cm}^{3}$ inoculè à un ơ de $23 \mathrm{~g}$.

"L'avant-bras" de la patte antérieure droite est enflé et paralysé, les doigts recroquevillés. Le crapaud marche en boitant comme s'il avait une patte raide. $6 \mathrm{~h}$ après il réagit peu et respire difficilement. Le lendemain il est vif, mais boite toujours avec sa patte raide et enflée. 3 jours après il meurt.

\section{40 glandes dans $0,25 \mathrm{~cm}^{3}$ inoculé à un ơ de $22 \mathrm{~g}$.}

I a solution a été inoculée aussitôt après sa préparation, aussi le venin n'était peut-être pas entièrement dissous. L'envenimation fut la même que dans le cas précédent, mais ce crapaud resta toujours plus vif. 4 jours après, j'ai constaté la mort.

3. 30 glandes dissoutes dans $0,4 \mathrm{~cm}^{3}$ inoculé à un ô de $19 \mathrm{~g}$.

Mêmes enflures, mais le crapaud réagit vivement 3 heures après et finalement survivra.

4. 10 glandes dans $0.5 \mathrm{~cm}^{3}$ inoculé à un ô de $20 \mathrm{~g}$.

Mêmes phénomènes d'envenimation, mais moins accentués. L'animal survit.

Lot tímoin : $0,6 \mathrm{~cm}^{3}$ d'eau physiologique inoculés à 3 crapauds de $20 \mathrm{~g}$.

Une légère enflure très passagère est observée. Il n'y a aucun boitillement et les crapauds conservent l'usage complet de leurs pattes.

\section{Troisième série d'expériences}

Cinq cents glandes ì venin extraites d'abeilles vivantes furent dissoutes dans $20 \mathrm{~cm}^{3}$ d'eau distillée. La solution obtenue servit plusieurs fois ; entre-temps elle était conservée à $-30^{\circ}$. On peut se demander alors si une telle solution garde intacte la virulence du venin qu'elle contient.

C. PiIssaltx (I922) affirme que la piqûre directe a toujours une action plus vive que le venin dissous et écrit : "Alors qu'une seule picuûre qui ne correspond plus qu'à $0,3 \mathrm{mg}$ de venin sec peu être mortelle pour une Souris (il y a des exceptions), l'inoculation de $0,5 \mathrm{mg}$ de venin dissous dans l'eau est insuffisante à déterminer la mort même par voie péritonéale."

Mes résultats corroborent ces chiffres. L'inoculation de $0,05 \mathrm{~cm}^{3}$, soit l'équivalent de $\mathrm{I}, 25$ glande fut mortelle par voie péritonéale pour I Souris sur 4 expérimentées (mort 2 jours après). $0,1 \mathrm{~cm}^{2}$. soit 2,5 glandes, est mortel, la prostration est immédiate, la respiration devient rapidement haletante ; le lendemain, le comportement semble redevenir normal, mais le $2^{\mathrm{e}}$ ou $3^{\mathrm{e}}$ jour la Souris meurt.

Par voie intraveineuse, l'envenimation d'une Souris est foudroyante, $0,1 \mathrm{~cm}^{3}$ tue une Souris de $27 \mathrm{~g}$ en 12 minutes, avec de très fortes convulsions, et $0,05 \mathrm{~cm}^{3}$ la tue en $\mathrm{I} \mathrm{h} \mathrm{Io}$. 
Résultats

\section{5 crapauds d'environ $20 \mathrm{~g}$.}

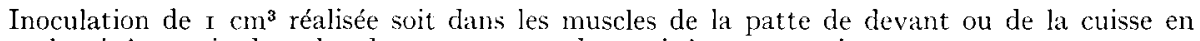
une seule piqûre, soit dans les deux pattes en deux picûres successives.

J'ai constaté la même paralysie qu'aux séances précédentes, celle des pattes de devant étant toujours plus durable et plus prononcée que celle des cuisses. Une légère enflure est encore consta. table pendant plusieurs semaines, mais les crapauds ne boitent plus.

Lot tímoin : des crapauds de même poids auxquels fut inoculée la même quautité d'eau distillée présentèrent la même enflure et un boitillement très passager.

\section{4 crapauds of de $20 \mathrm{~g}$.}

Inoculation de $\mathrm{I} \mathrm{cm}^{3}$ dans la langue et les flancs. 2 bavèrent après quelques heures. 3 moururent les jours suivants.

\section{4 crapauds of de $50,60,80$ et $100 \mathrm{~g}$.}

a) Inoculation de $\mathrm{cm}^{3}$ dans la patte antérieure pour l'un et dans les flancs pour un deuxième. Celui qui fut piqué aux flancs ne chassa plus, il avait une sorte d'œedène sous-cutané. Le lendemain, il se remit à chasser. L'autre avait sa patte antérieure droite enfléc et paralysée. mais le comportement de chasse ne se modifia nullement.

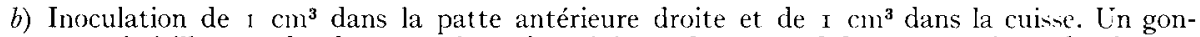
flement et un boitillement des deux membres s'ensuivirent. Le crapaud de $80 \mathrm{~g}$ continua de chasser, tandis que celui de $50 \mathrm{~g}$ avait peur des proies qui lui étaient présentées.

De toutes ces expériences, on peut conclure que la résistance de Bufo bufo au venin d'Apis mellifica est vraiment très forte.

Je peux donc écrire que si l'injection de l'équivalent de 1,25 glandes par voie intraveineuse ou de 2,5 glandes par voie péritonéale suffisent à tuer une Souris de $30 \mathrm{~g}$, il faut, par contre, l'équivalent de 30 à 40 glandes par voie musculaire pour obtenir la mort d'un Bufo bufo de même poids ( 25 glandes dans les flancs ou la langue peuvent arriver au même résultat). Quant aux Crapauds, de plus de $50 \mathrm{~g}, 2 \mathrm{~cm}^{3}$, soit l'équivalent de $5^{\circ}$ glandes, n'ont pas d'effet mortel par voie musculaire, ils sont donc plus résistants à l'action du venin.

Il est évident que dans la nature, des injections de pareilles doses ne sont jamais atteintes par des piqûres d'Abeilles. Des crapauds adultes de tout poids (20 à Ioo g) piqués de Io à 18 fois ne présentèrent pas de phénomènes d'envenimation. Les résultats de cette petite expérience complémentaire expliquent donc que, malgré la douleur momentanée occasionnée par une ou plusieurs piqûres dans la gorge, le Crapaud continue à chasser les Abeilles ou tout au moins s'en écarte pour peu de temps.

Les Crapauds étant très résistants à l'action du venin, la grande diversité des réactions individuelles (cf tabl. I) provient autant du degré de sensibilité au venin et à la piqûre que de l'habileté à saisir une proie et de la vitesse à l'avaler. Généralement, ces caractères sont liés à la taille et donc à l'âge du Crapaud. De grosses femelles comme le $n^{\circ}$ II $\left(D_{1}\right)$ sont vraiment peu sensibles aux piqûres. L'intensité des réactions dépend aussi du facteur individuel de l'Abeille, son degré d'agressivité, la virulence de son venin et la rapidité de sa défense.

En définitive 1'expérimentateur ne peut pas noter toutes les piqûres "internes" puisque certaines ne se manifestent par aucune réaction de "malaise " mais il pourra enregistrer les piqûres internes " douloureuses " les plus importantes, car elles influenceront le comportement prédateur. 
Quant aux piqûres externes, elles sont faciles à observer. Généralement elles provoquent la fuite. Contrairement à Cơ'T, j'en ai noté très peu, cela tient à l'heure de mes expériences et à la faible agressivité de mes ruches.

\section{d) Importance des dates de l'expérience.}

Une autre constatation s'impose aussi à l'expérimentateur : c'est que, malgré le caractère automatique des piqûres internes, le nombre de ces piqûres tant internes qu'externes dépend finalement en grande partie de l'agressivité de la ruche. Or, si celle-ci peut changer beaucoup d'une ruche à l'autre (LECOMTE, I96I), elle varie aussi avec le temps. Pour une même ruche le degré d'agressivité est presque nul en période de grande miellée (printemps, mai, juin, ex : série $\mathrm{D}$ et $\mathrm{A}^{\prime \prime}$ ), mais il sera très grand au moment des pillages (ex. : juillet I96I série B, série $A^{\prime \prime \prime}$ ). Pour $\mathrm{L}_{\mathrm{E}} \mathrm{COMTE}$ l'influence du vent et de l'orage est certaine ; je l'ai constaté en juillet Ig62 sur les séries $\mathrm{E}$ et $\mathrm{D}$. Les autres conditions atmosphériques (pluie, froid) perturbent aussi l'activité des ruches (série $\mathrm{C}$ et $\mathrm{A}^{\prime}$ ).

\section{2. - MÉTHOde}

Comme dans les expériences de Cort, le Crapaud, à jeun depuis plusieurs jours, était posé devant l'entrée de la ruche sur une plate-forme située à son niveau. Parfois cette plate-forme existait déjà, c'était la planche d'envol de la ruche, légèrement inclinée ou horizontale (série $\mathrm{C}, \mathrm{A}, \mathrm{A}^{\prime \prime}$ ), je l'agrandissais au besoin pour l'expérience. Ces plates-formes étaient généralement à 30 ou $40 \mathrm{~cm}$ du sol; le Crapaud " hésitait "à sauter durant les premières séances. Le Crapaud pouvait donc se nourrir de lui-même sans interruption jusqu'à ce qu'il s'en aille, en sautant de la plate-forme.

A Bures-sur-Yvette (série $A^{\prime \prime \prime}$ et B) les ruches n'étaient pas surélevées et la sortie des Abeilles était de plain-pied avec les soubassements en ciment. Un cadre à trois côtés encastrait le devant de la ruche, empêchant le Crapaud de s'éloigner aussitôt.

A partir des premières observations décrites plus haut, j'ai décidé de modifier certaines conditions expérimentales de CotT et d'employer les méthodes suivantes:

a) Opérer le soir et à la tombée de la nuit.

Avec plus ou moins de diversité, les expériences se sont déroulées au crépuscule à la nuit complète. J'ai même choisi des heures tardives $(2 \mathrm{I} h$ à $23 \mathrm{~h}$ ) pour les dernières séries $(\mathrm{D}, \mathrm{E}, \mathrm{F})$. La série A fait donc exception avec ses heures matinales ou de fin d'après-midi. Je l'ai quand même incluse dans mes statistiques pour faire la comparaison avec COTT et à cause des essais ultérieurs avec les mêmes crapauds.

Tout ceci avait pour conséquence :

$I^{0}$ De supprimer les vols d'abeilles qui effrayaient les crapauds,

$2^{\circ}$ De réduire l'activité de la ruche et donc de diminuer son agressivité.

Il restait suffisamment de déplacements de ventileuses ou de gardiennes isolées ; plus tôt, les abeilles ne sont pas approchées par les crapauds car elles sont encore agglomérées en paquet au bord de la ruche. J'attendais pour commencer l'expérience que les vols eussent quasiment cessé et que la ruche fût calme.

\section{b) Utiliser des Crapauds adultes de toute taille.} gereuse.

J'ai voulu spécialement savoir si de gros Bufo bufo parvenaient à s'habituer à cette proie dan-

c) Réaliser les expériences sur 9 jours au lieu de $\%$.

Pour Сотт, au bout de 7 jours, plus aucun Crapaud ne mangeait d'Abeilles. En prolongeant l'expérience ( 9 à I 5 jours), je me suis rendu compte que les Crapauds recommençaient à manger. J'ai choisi une durée de 9 jours pour garder une marge de vérification aux résultats positifs du $7^{\mathrm{e}}$ jour. 
d) Compter une piqûre interne (gorge ou estomac) "douloureuse " chaque fois que le crapaud après avoir happé une abeille, manifestait des signes caractéristiques de " malaise ".

Les piqûres internes sont peut-être plus nombreuses, mais finalement ce sont les plus douloureuses, celles qui causent ces " crispations " qui feront modifier le comportement prédateur de Bufo bufo vis-à-vis des Abeilles.

\section{TABLEAU 2}

Dates d'expériences

\begin{tabular}{|c|c|c|c|c|c|}
\hline \multirow[t]{4}{*}{ Séries 1961} & & & $1^{\text {er }}$ essai $\left(A^{\prime}\right)$ & $2^{\mathrm{e}}$ essai $\left(\mathrm{A}^{\prime \prime}\right)$ & $3^{\mathrm{e}}$ essai ( $\left.\mathrm{A}^{\prime \prime \prime}\right)$ \\
\hline & Série A & $\begin{array}{l}A_{1} \\
A_{2} \\
A_{3} \\
A_{4} \\
A_{5}\end{array}$ & $\begin{array}{l}22-5-61-30-5-61 \\
22-5-61-30-5-61 \\
22-5-61-30-5-61 \\
22-5-61-30-5-61 \\
22-5-61-30-5-61\end{array}$ & $\begin{array}{l}18-6-61-26-6-61 \\
20-6-61-28-6-61 \\
14-6-61-22-6-61 \\
14-6-61-22-6-61 \\
1 \times-6-61-22-6-61\end{array}$ & $\begin{array}{l}21-7-61-29-7-61 \\
21-7-61-29-7-61 \\
21-7-61-29-7-61 \\
21-7-61-29-7-61\end{array}$ \\
\hline & Série B & $\begin{array}{l}\mathrm{B}_{1} \\
\mathrm{~B}_{2}\end{array}$ & $\begin{array}{l}21-7-61-29-7-61 \\
22-7-61-30-7-61\end{array}$ & & \\
\hline & Série C & $\begin{array}{l}\mathrm{C}_{1} \\
\mathrm{C}_{2} \\
\mathrm{C}_{3} \\
\mathrm{C}_{4}\end{array}$ & $\begin{array}{l}6-10-61-16-10-61^{*} \\
6-10-61-16-10-61 \\
6-10-61-16-10-61 \\
6-10-61-16-10-61\end{array}$ & & \\
\hline \multirow[t]{2}{*}{ Séries 1962} & Série D & $\begin{array}{l}\mathrm{D}_{1} \\
\mathrm{D}_{2} \\
\mathrm{D}_{3} \\
\mathrm{D}_{4} \\
\mathrm{D}_{5} \\
\mathrm{D}_{6} \\
\mathrm{D}_{7} \\
\mathrm{D}_{8} \\
\mathrm{D}_{9} \\
\mathrm{D}_{10}\end{array}$ & $\begin{array}{r}1^{\text {er }} \text { essai }\left(D^{\prime}\right) \\
16-6-62-2{ }^{4}-6-62 \\
16-6-62-24-6-62 \\
17-6-62-25-6-62 \\
2-6-62-10-6-62 \\
10-6-62-18-6-62 \\
10-6-62-18-6-62 \\
11-6-62-19-6-62 \\
2-6-62-10-6-62 \\
15-6-62-23-6-62 \\
2-6-62-10-6-62\end{array}$ & $\begin{array}{c}2^{\mathrm{e}} \text { essai }\left(\mathrm{D}^{\prime \prime}\right) \\
7-7-62-15-7-62 \\
7-7-62-15-7-62\end{array}$ & \\
\hline & Série E & $\begin{array}{l}E_{1} \\
E_{2} \\
E_{3} \\
E_{4} \\
E_{5}\end{array}$ & $\begin{array}{l}5-7-62-13-7-62 \\
5 \cdot 6-62-13-7-62 \\
6-7-62-14-7-62 \\
5-7-62-13-7-62 \\
6-7-62-14-7-62\end{array}$ & & \\
\hline Série 1963 & Série F & $\begin{array}{l}\mathrm{F}_{1} \\
\mathrm{~F}_{2} \\
\mathrm{~F}_{3} \\
\mathrm{~F}_{4}\end{array}$ & $\begin{array}{l}12-7-63-20-7-63 \\
12-7-63-20-7-63 \\
12-7-63-20-7-63 \\
12-7-63-20-7-63\end{array}$ & & \\
\hline
\end{tabular}

Une pluie continuelle les 7-10-61 et 9-10-61 reporta les $2^{\mathrm{e}}$ et $3^{\mathrm{e}}$ séances $\left(2^{\mathrm{e}}\right.$ et $3^{\mathrm{e}}$ jours) au 8-10-61 et au 10-10-61.

e) Pratiquer comme CoTT un étalement des dates d'expériences pour éliminer l'effet des conditions atmosphériques sur le déroulement des expériences et l'activité des crapauds et des abeilles.

La pluie perturbe les sorties des abeilles ; par deux fois elle empêcha l'expérience du $7^{\mathrm{e}}$ jour de se dérouler normalement. C'est pourquoi j'opérais parfois un décalage d'un ou deux jours dans les dates d'expériences d'une même série (cf. tabl. 2). Bien que mes préférences aillent aux périodes les 
plus propices aux crapauds, les expériences, étalées sur plusieurs mois et trois années consécutives, sont assez nombreuses et assez diverses pour que leur bilan puisse se comparer aux résultats de CoTT et présente une valeur certaine de généralité.

\section{3. - MATÉRIEL}

Abeilles. - $1^{\circ}$ Rucher situé dans un verger à Chevilly-Larue (Seine). Plusieurs ruches de constructions légèrement différentes, peuplées d'Abeilles communes à abdomen noir, peu agressives, furent utilisées par les séries $\mathrm{A}^{\prime}, \mathrm{A}^{\prime \prime}, \mathrm{C}, \mathrm{D}, \mathrm{D}^{\prime \prime}, \mathrm{E}$ et $\mathrm{F}$ et diverses expériences complémentaires.

$2^{\mathrm{o}}$ Rucher de la station apicole de Bures-sur-Yvette (Essonne). Les ruches situées à même le sol sont peuplées d'Abeilles beaucoup plus agressives, spécialement les races qui furent utilisées pour l'expérience (race italienne, et une variété du Tafilalet).

Crapauds : 30 Bufo bufo adultes furent utilisés. 15 d'entre eux mesuraient de 9 à $12 \mathrm{~cm}$ et pesaient plus de $100 \mathrm{~g}$, tandis que les $\mathrm{I} 5$ autres mesuraient de 6 à $9 \mathrm{~cm}$ et pesaient moins de $\mathrm{I} 00 \mathrm{~g}$ (il y a deux exceptions pour les poids de $A_{2}$ et de $E_{1}$ ). (Cf. tabl. 37 , et tabl. 7 p. 108.)

TABLEAU 3

Taille et poids des Crapauds

\begin{tabular}{|c|c|c|c|c|c|}
\hline & $(\mathrm{mm})$ & $(\mathrm{g})$ & & $(\mathrm{mm})$ & $(\mathrm{g})$ \\
\hline $\begin{array}{c}\text { Série A } \\
\mathrm{A}_{1} \\
\mathrm{~A}_{2} \\
\mathrm{~A}_{3} \\
\mathrm{~A}_{4} \\
\mathrm{~A}_{5} \\
\text { Série } B \\
\mathrm{~B}_{1} \\
\mathrm{~B}_{2} \\
\text { Série } C \\
\mathrm{C}_{1} \\
\mathrm{C}_{2} \\
\mathrm{C}_{3} \\
\mathrm{C}_{4} \\
\text { Série } F \\
\mathrm{~F}_{1} \\
\mathrm{~F}_{2} \\
\mathrm{~F}_{3} \\
\mathrm{~F}_{4}\end{array}$ & $\begin{array}{r}112 \\
93 \\
66 \\
68\end{array}$ & $\begin{array}{r}115 \\
102 \\
87 \\
58 \\
45\end{array}$ & $\begin{array}{c}\text { Série } D \\
\mathrm{D}_{1} \\
\mathrm{D}_{2} \\
\mathrm{D}_{3} \\
\mathrm{D}_{4} \\
\mathrm{D}_{5} \\
\mathrm{D}_{6} \\
\mathrm{D}_{7} \\
\mathrm{D}_{8} \\
\mathrm{D}_{9} \\
\mathrm{D}_{10} \\
\text { Série E } \\
\mathrm{E}_{1} \\
\mathrm{E}_{2} \\
\mathrm{E}_{3} \\
\mathrm{E}_{4} \\
\mathrm{E}_{5}\end{array}$ & $\begin{array}{r}115 \\
110 \\
110 \\
106 \\
110 \\
102 \\
102 \\
105 \\
92 \\
92\end{array}$ & $\begin{array}{l}220 \\
195 \\
167 \\
130 \\
155 \\
135 \\
140 \\
155 \\
125 \\
105\end{array}$ \\
\hline
\end{tabular}

III. - RÉSULTATS

L,e tableau 4 résume toutes les expériences ( ${ }^{\text {er }}$ essai) effectuées avec les 30 crapauds en I96I, I962 et I963. Il a été constitué avec les mêmes sigles que CoTT pour établir une comparaison plus facile avec ses résultats (cf. son tableau 2, Proc. Zool Soc. I936, p. II5), 
Somm

\begin{tabular}{|c|c|c|c|c|c|}
\hline $\mathrm{N}^{\circ}$ & $1^{\text {er }}$ jour & $2^{e}$ jour & $3^{e}$ jour & $4^{e}$ jour & $5^{e}$ jour \\
\hline $\begin{array}{l}196 \mathrm{l} \\
\mathrm{A}_{1} \\
\mathrm{~A}_{2} \\
\mathrm{~A}_{3} \\
\mathrm{~A}_{4} \\
\mathrm{~A}_{\overline{5}}\end{array}$ & $\begin{array}{l}1 * \\
1 * \\
1,1\end{array}$ & $\begin{array}{l}1,1^{*} \\
1^{*}, 1^{*}, 1,1^{*} \\
1^{*} \\
1^{*}, 1^{*} \\
1^{*}\end{array}$ & $\begin{array}{l}1^{*}, 1^{*}, 1^{*}, 1,1^{*}, 1, \\
1^{*} \\
1,1^{*}, 1 \\
1^{*}, 1,1,1,1^{*} \\
1^{*}\end{array}$ & $\begin{array}{l}1,1^{*}, 1^{*}, 1 \\
1^{*}, 1^{*}, 1,1,1,1,1 \\
1^{*}\end{array}$ & $1,1^{*}, 1^{*}$ \\
\hline $\begin{array}{l}\mathrm{B}_{1} \\
\mathrm{~B}_{2}\end{array}$ & $1,1^{*}$ & $1,1,1,1,1,1,1$ & $\begin{array}{l}2,1,1,1,1,1,1,1 \\
\quad 1,1,1,1\end{array}$ & $1,1^{*}, 1$ & 1,1 \\
\hline $\begin{array}{l}\mathrm{C}_{1} \\
\mathrm{C}_{2} \\
\mathrm{C}_{3} \\
\mathrm{C}_{4}\end{array}$ & & & $\begin{array}{l}1,1^{*}, 1^{*}, 1,1^{*}, 1 \\
1,1^{*}, 1,1^{*}\end{array}$ & $1^{*}, 1,1,1,1^{*}, 1$ & $1^{*}, 1,1,1,1,1^{*}$ \\
\hline $\begin{array}{c}1962 \\
\mathrm{D}_{1} \\
\end{array}$ & $1,1,1,1,1,1,1,1$ & $1,1,1$ & $1,1,1,1,1,1,1^{*}, 1$ & - & $\begin{array}{r}1,1,1,1,1,1,1 \\
1,1,1,1,1,1\end{array}$ \\
\hline $\begin{array}{l}\mathrm{D}_{2} \\
\mathrm{D}_{3} \\
\mathrm{D}_{4} \\
\mathrm{D}_{5} \\
\mathrm{D}_{6} \\
\mathrm{D}_{7} \\
\mathrm{D}_{8} \\
\mathrm{D}_{9} \\
\mathrm{D}_{10}\end{array}$ & $\begin{array}{l}1,1,1,1,1,1,1,1 \\
\quad 1,1,1,1 \\
1,1,1,1,1 \\
1,2^{*} \\
1,1,1,1,1 \\
1^{*} \\
1\end{array}$ & $\begin{array}{l}1,1,1,1,1,1,1 \\
1,1,1,1,1 \\
1,1,1,1,1,1^{*}, 1,1,1 \\
1,1,1,1,1,1,1,1^{*} \\
1,1,1,1,1,1^{*} \\
2,1,1,1^{*} 1,1,1,1,1^{*} \\
1,1,1,1, \rightarrow 22 \mathrm{Ab} \\
1,1,1\end{array}$ & $\begin{array}{l}1,1,1,1,1,1,1,1^{*} \\
1,1,1,1,1,1,1,1 \\
1^{*} \\
1,1 \\
2 \\
1,1,1,1^{*}, 1^{*}, 1,1 \\
1,1,1 \\
1,1,1,1^{*}\end{array}$ & $\begin{array}{l}1,1,1,1^{*}, 1,1,1 \\
1^{*} \\
1^{*}, 1,1,1^{*} \\
1^{*} \\
1 \\
1\end{array}$ & $1,1,1,1$ \\
\hline $\begin{array}{l}E_{1} \\
E_{2} \\
E_{3} \\
E_{4} \\
E_{5}\end{array}$ & $\begin{array}{r}1,1,1,1^{*}, 1,1,1 \\
1,1,+ \\
1,1,1,1,1,1,1^{*} \\
1,1^{*}, 1^{*}, 1,1^{*}, 1^{*}, \\
1,1,1,1^{*}, 1,1,1 \\
1,1,1,1,1,1^{*} 1, \\
1,1^{*}, 1^{*}, 1,1,1 \\
1^{*}, 1^{*}, 1,1^{*}\end{array}$ & $1,1 *$ & 1 & $\left\{\begin{array}{l}1,1^{*} \\
1,1^{*}\end{array}\right.$ & 1* \\
\hline $\begin{array}{l}1963 \\
F_{1} \\
F_{2} \\
F_{3} \\
F_{4}\end{array}$ & $\begin{array}{l}1,1,1 \\
1,1,1 \\
1\end{array}$ & $\begin{array}{l}1,1 \\
1,1 \\
1\end{array}$ & $1_{1 *}^{1,1,1^{*}}$ & $\begin{array}{l}1 \\
1\end{array}$ & $\mid \begin{array}{l}1,1 \\
1 \\
1 \\
1\end{array}$ \\
\hline & $119 \mathrm{Ab}$. & 87 & 88 & 43 & 43 \\
\hline
\end{tabular}


" I " ou " 2 " signifie qu'une ou deux abeilles ont été avalées par un coup de langue.

(*) signifie que le crapaud a manifesté une réaction de "malaise " à la suite d'une piqûre interne douloureuse,

$(+)$ signale une piqûre externe.

(-) indique que ce jour là, le crapaud n'a pas mangé parce qu'il muait.

3 essais furent réalisés avec les crapauds de la série A (tabl. 5), ils indiquent que les mêmes individus ne réagissent pas de la même façon lorsque les conditions d'expérience changent.

\section{TABLEAU 5}

Nombre d'abeilles avalées par les crapauds de la série A pendant leurs 3 essais

\begin{tabular}{|c|c|c|c|c|c|c|c|c|c|c|c|}
\hline & Crapauds & fer jour & 2e jour & 3e jour & $4^{e}$ jour & $5^{e}$ jour & $6^{\text {e jour }}$ & $7^{\text {e jour }}$ & se jour $^{-}$ & ye jour & Total \\
\hline & $1^{\text {er }}$ essai $\left(A^{\prime}\right)$ & i & & & & & & & & & \\
\hline$A_{1}$ & $\ldots \ldots \ldots \ldots \ldots$ & 1 & 2 & 7 & 4 & 3 & 2 & () & 1 & 't & 品 \\
\hline$A_{2}$ & $\ldots \ldots \ldots \ldots \ldots$ & 0 & '́ & 3 & 7 & 0 & 1 & 0 & 0 & 0 & 15 \\
\hline$A_{3}$ & $\ldots \ldots \ldots \ldots \ldots$ & 1 & 1 & 0 & 1 & 0 & 0 & 0) & 0 & 0 & 3 \\
\hline$A_{4}$ & $\ldots \ldots \ldots \ldots \ldots$ & 1 & 2 & 5 & 0) & 0 & 0 & 0 & 0 & 0 & 8 \\
\hline$A_{5}$ & $\ldots \ldots \ldots \ldots$ & 2 & 1 & 1 & 0 & 0 & 0 & 0 & 0 & 0 & 't \\
\hline & 2 essai $\left(A^{\prime \prime}\right)$ & & & & & & & & & & \\
\hline$A_{1}$ & $\ldots \ldots \ldots \ldots$ & 4 & 8 & 8 & 's & 9 & 1 & 4 & i) & 2 & 46 \\
\hline$A_{2}$ & $\ldots \ldots \ldots \ldots$ & 8 & 1 & 0 & '́ & 8 & 5 & 5 & 10 & 3 & $4 ' t$ \\
\hline$A_{3}$ & & 13 & 0 & 1 & 1 & 1 & 2 & 0) & 0 & 2 & 20 \\
\hline$A_{4}$ & $\ldots \ldots \ldots \ldots \ldots$ & 0 & 0 & 0 & 0 & 1 & 3 & 2 & 0 & 1 & 7 \\
\hline$A_{5}$ & $\ldots \ldots \ldots \ldots$ & 1 & 1 & 0 & 0 & 0 & 0 & 0) & 0 & 0 & 3 \\
\hline & $3^{\mathrm{e}}$ essai $\left(\mathrm{A}^{\prime \prime \prime}\right)$ & & & & & & i & & & & \\
\hline$A_{1}$ & $\ldots \ldots \ldots \ldots \ldots$ & 4 & 9 & 9 & 2 & 1 & 8 & 0 & () & () & 33 \\
\hline$A_{2}$ & $\ldots \ldots \ldots \ldots$ & 3 & 1 & 0) & 2 & 0 & 0 & 1) & 0 & 0) & 6 \\
\hline$A_{4}$ & $\ldots \ldots \ldots \ldots$ & 1 & 1 & 0 & 0 & 0 & 1 & 0 & 2 & 0 & 5 \\
\hline$A_{5}$ & $\ldots \ldots \ldots \ldots$ & 2 & 0 & 0 & 0 & 1 & 1 & 0 & 0 & 1 & 5 \\
\hline
\end{tabular}

\section{IV. - COMMENTAIRE DES RÉSULTATS}

\section{Absence de refus instinctif}

Il n'y a pas de refus instinctif de l'Abeille de la part du Crapaud commun (cf. tabl. 4 et fig 3). Au contraire, l'Abeille "attire "l'attention du Crapaud. Ceci s'explique facilement par les lois du comportement prédateur de Bufo bufo. En effet ce comportement inné est uniquement déclenché par le mouvement de la proie (une proie immobile n'est jamais avalée). Ce " mouvement " est repéré visuellement et quelquefois tactilement; il n'est pas un stimulus élémentaire mais la résultante de plusieurs stimuli secondaires qui par effet additif augmentent son efficacité : ces composantes du mouvement sont sa vitesse, sa forme et sa direction ( $\mathrm{L}_{4}$ ESCURE, I965). Le mode de déplacement d'A pis mellifica à l'entrée de la ruche (mouvement de marche) et sa vitesse correspondent aux meilleurs de ces stimuli. 
De même, au-delà d'une certaine taille, variable avec la taille du prédateur, le mouvement de la proie ne provoque plus de réaction alimentaire mais un comportement de fuite. Sous ce rapport, la dimension de l'Abeille est optima et il est certain que ses couleurs vives et alternées doivent faciliter sa découverte. L'Abeille se déplaçant à l'entrée de sa ruche est donc un excellent stimulus-signal pour l'appétit du Crapaud.

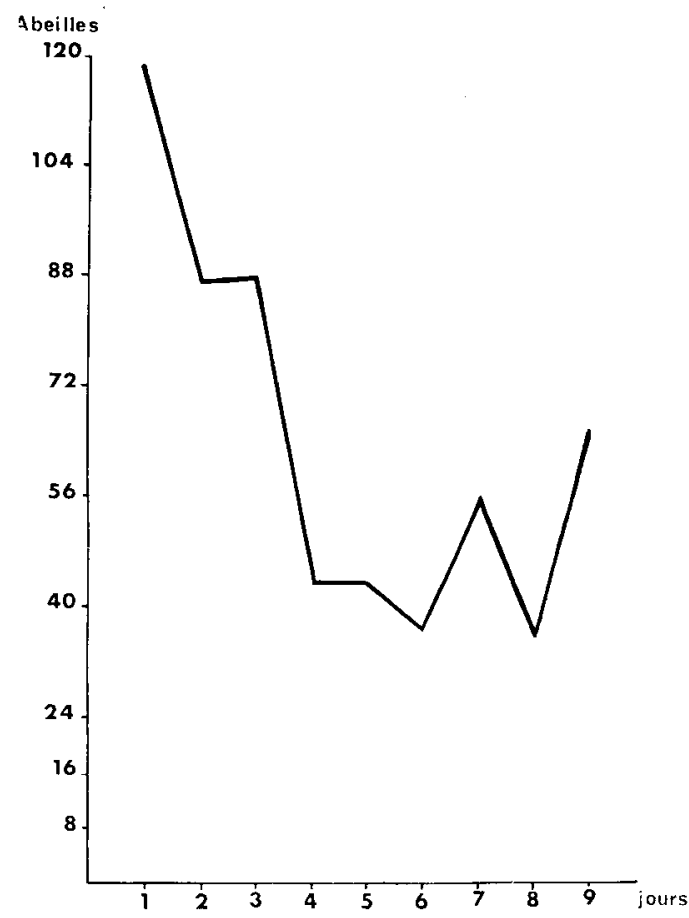

FIG. 3. - Nombre d'abeilles avalées chaque jour par les 30 crapauds

C'est en constatant l'absence de répugnance instinctive du Crapaud vis-à-vis de l'Abeille, durant ses expériences, que Cotr (I936, I940) a pu affirmer que la répulsion envers des proies aposématiques est acquise et non innée.

Pourquoi, alors, la réponse à des stimuli aussi normaux fut-elle bloquée, le premier jour, chez Io crapauds? Il faut en voir la cause dans le "dépaysement", le changement de décor et, à un moindre degré, le trouble que peut provoquer la manipulation par l'expérimentateur. On pourrait ajouter aussi qu'une abeille devant sa ruche est plus agressive que dans un vivarium. Cette différence s'atténue beaucoup lorsque les vols ont cessé et que la ruche est calme; $\mathrm{D}_{3}, \mathrm{D}_{4}$ et $\mathrm{D}_{7}$ fuyaient une planche d'envol où les abeilles ne montraient pourtant aucune agressivité. Le cas de la série $C$ est particulier ; il est dû en grande partie à la pluie continuelle des premiers jours, qui, malgré les moments de relative accalmie où j'ai réalisé mes essais, a perturbé l'activité de la ruche et le comportement du crapaud.

Après cette abstention du premier jour, des réactions prédatrices normales apparurent les jours suivants chez ces Io crapauds. Avec plus ou moins de retard, 
ils rejoignirent donc le cas général et manifestèrent ensuite les mêmes modifications de comportement que leurs congénères.

\section{Modification du comportement prédateur du Crapaud vis-à-vis des Abeilles}

Sa répulsion pour l'Abeille étant acquise et non innée, Bufo bufo apprend-ilà refuser ces Hyménoptères? CỡT (I936) dans la discussion de ses expériences, décrit et compare l'attitude devant la ruche du Crapaud inexpérimenté et du Crapaud " ayant appris sa leçon ". J'ai également constaté une modification du comportement de mes crapauds. Dans bien des cas, elle est spectaculaire; mais (et en cela je ne suis pas d'accord avec Cor'T) elle peut aussi bien aller dans le sens d'une acceptation que d'un refus de la proie. En définitive, elle dépend du nombre de piqûres "douloureuses" subies, qui est lui-même fonction de l'agressivité đes Abeilles et de l' " habileté "du Crapaud.

En général, à leur première séance, les crapauds commençaient par rester immobiles à l'endroit où l'expérimentateut les avait posés. Après 30 secondes ou une minute, ils tournaient la tête vers les abeilles. Le plus souvent, c'était une abeille isolée passant près d'eux qui avait déclenché les réactions alimentaires. Ils avançaient alors avec des gestes saccadés et lançaient la langue sans toujours attendre le meilleur moment : l'abeille visée était souvent soit trop loin, soit agglomérée à d'autres abeilles. Les signes de " malaise » se manifestaient aussitôt (piqûres) mais le crapaud continuait à guetter; les premières abeilles étaient avalées toutes les minutes, puis l'intervalle de temps entre deux captures augmentait... Le crapaud moins tendu, n'était plus dressé sur ses pattes. Il guettait de plus en plus longuement avant de happer, puis finalement quittait l'entrée de la ruche et s'en allait.

Plusieurs crapauds (Série E) manifestèrent le premier jour une précipitation, que je juge caractéristique, d'une faim intense. Aussitôt placés, les crapauts s'approchaient de l'amas d'abeilles qui se trouve toujours à l'entrée d'une ruche. Dressés sur les pattes, le cou tendu, ils s'arrêtaient près des abeilles et lançaient leur langue aussitôt. Avec un tel procédé, les piqûres ne se faisaient pas attendre, elles ne ralentissaient pas l'ardeur du prédateur; ils avalaient même les abeilles qui grimpaient sur leur bouche, se faisaient piquer à chaque fois.

De gros Bufo bufo qui avaient subi le même temps de jeûne que leurs congénères ne montrèrent pas cette précipitation, néfaste aux crapauds, puisqu'elle augmente les risques de piqûres. Ils s'approchaient de l'entrée de la ruche, se mettaient sur le côté et guettaient les abeilles qui sortaient d'un orifice de la ruche. Ils visaient seulement les abeilles isolées, négligeant celles qui ne débouchaient pas devant eux $\left(\mathrm{D}_{1}\right.$, $\mathrm{D}_{2} ; \mathrm{A}_{1}, \mathrm{~A}_{2}, 2^{\mathrm{e}}$ essai). L'immobilité durant l'affît, leur faisait éviter les attaques des abeilles ; ils ne se dressaient pas sur leurs pattes pour happer, mais, en s'aplatissant, ils tendaient le cou et exécutaient un léger mouvement de balancier en projetant leur langue.

Après leur premier contact avec les Abeilles, comment se comportent les Crapauds? Il est certain que les jours suivants, leur comportement prédateur subit une modification et la lecture du graphique de la figure 3 nous indique nettement une diminution de la consommation d'abeilles. Cette diminution est progressive et s'accentue, surtout à partir du troisième jour. Je pense d'ailleurs que le maximum du premier jour est un peu artificiel, à cause de l'excitation due au jeûne prolongé. Pour 
la série $F$ dont les crapauds ont jeûné moins longtemps, le maximum est étalé sur les trois premiers jours.

Le comportement lui-même se modifie plus rapidement que la consommation. Dès le deuxième jour, le Crapaud adopte une attitude nouvelle en face de la ruche. La précipitation du premier jour s'est évanouie : il s'approche lentement de l'entrée, se tient immobile, guette et attend le passage devant lui d'abeilles isolées pour lancer sa langue. Il s'éloigne du lieu où les abeilles sont nombreuses ou agglomérées. Si une abeille lui grimpe sur la tête ou sur la bouche, il réagit seulement en fermant les yeux. S'il se fait piquer en avalant une abeille, il quitte la ruche tout de suite ou après une autre tentative. Les jours suivants, ces modifications subsistent ou s'accentuent.

Les uns continuent à guetter, avalent quelques abeilles, se font piquer et s'en vont aussitôt après $\left(\mathrm{F}_{1}, \mathrm{~F}_{1}, \mathrm{~F}_{2}, \mathrm{~A}_{1}, \mathrm{I}_{10}\right)$. I,es autres quittent la plate-forme sitôt qu'ils y sont placés, sans avoir guetté, ni regardé les abeilles. Ce manège peut durer plusieurs jours, mais bien souvent ils recommencent à guetter et à avaler des abeilles après un intervalle de quelques jours. Certains crapauds font exception et révèlent plutôt une adaptation positive à cette proie dangereuse. Avec quelques petites fluctuations, ils continuent à capturer leur ration quotidienne d'abeilles. Comme nous l'avons dit plus haut, quelques-uns manifestèrent même cette adaptation dès le premier jour ; ils n'eurent donc pas à changer ensuite leur attitude.

Sauf ces individus plus " habiles », les Crapauds modifient donc leur comportement envers les Abeilles; même s'ils continuent à les happer, ils ne les chassent jamais comme un Ver de farine ou un Pyrrhocoris. Le guet et le "temps d'arrêt (1) " devant la proie est long, le coup de langue ne part qu'à coup st̂̀r vers une abeille isolée.

\section{Absence de refus total}

Selon ce que nous venons de dire du changement de comportement de Bufo bufo vis-à-vis des Abeilles, l'ardeur à les poursuivre irait en diminuant. Aboutirait-on finalement à un refus total des Abeilles?

Fin fait, si 20 crapauds sur 30 ont mangé I I 9 abeilles le premier jour de l'expérience, I 8 , soit $60 \mathrm{p}$. IOo, en avalèrent encore 66 le neuvième jour. Malgré les piqûres, les crapauds continuaient ou recommençaient à avaler des abeilles. Je n'ai donc pas eu, avec cette proie, d'exemples de refus aussi nets que ceux obtenus par BUYTENDIJK (I9I8) avec Formica ruja et SchNeIdER (I954) avec le Ver à soie.

Pour dégager des résultats plus clairs, $\mathrm{j}$ 'aurais pu réaliser un dressage uniquement sur l'Abeille en effectuant l'expérience dans le vivarium d'un crapaud et non devant la ruche. Il suffit d'introduire les abeilles les unes après les autres dans le vivarium, mais nous procédons alors à une expérience très artificielle.

Ił'Abeille isolée n'est pas dans son élément naturel et n'a pas la même agressivité. En vivarium, l'Abeille est aussitôt poursuivie, facilement avalée sans signe de malaise dans la grande majorité des cas.

Dans la nature, le Crapaud a en face de lui l'Abeille-devant-sa-ruche. Même si la ruche n'est pas agressive, le changement de décor perturbe le comportement de cer-

( ${ }^{1}$ Lorsqu'un Crapaud s'approche d'une proie et arrive à portée de langue, il s'arrête et fixe la proie du regard. Ce "stop" pour mieux viser et lancer sa langue est toujours observé; il est plus ou moins bref suivant le degré de stimulation de la jroie (comportement inné) ou sa signification de pumition (comportement acquis). 
tains crapauds. Seuls, le nombre et la diversité des spécimens utilisés permet d'atténuer ce facteur qu'on ne peut pas supprimer pour chacun. De toute façon, avec l'Abeille, un Crapaud qui réagit normalement, a devant lui une proie ambivalente. Elle est comestible, car elle n'est pas recrachée. Si elle est avalée sans qu'il y ait piqûre, elle est bonne. Elle est signal de récompense, c'est-à-dire satisfaction du comportement alimentaire. S'il y a piqûre interne, l'Abeille devient signal de punition plus ou moins fort suivant la virulence du venin, l'emplacement de la piqûre et la résistance du Crapaud.

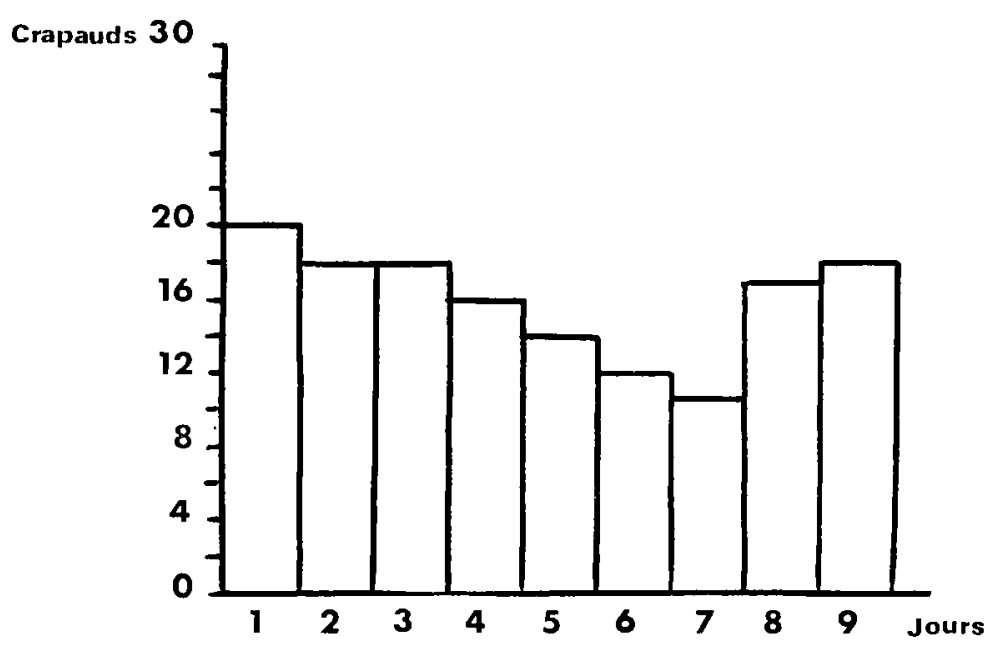

Fic. 4. - Nombre de crapauls avalant des abeilles chaque jour

A cause de cette double possibilité de punition et de récompense, on constate dans la consommation des abeilles par les crapauds des baisses momentanées, des refus temporaires, mais non définitifs (fig. 3 et 4 ). Comment interpréter ces variations?

Il est certain que, malgré sa nouveauté, la vue de l'Abeille provoque la formation d'un "potentiel excitatif " ( $\mathrm{L}_{\mathrm{E}} \mathrm{Ny}, \mathrm{I}$ 96I) de la réaction alimentaire (comportement inné). Si les crapauds ne sont pas piqués douloureusement $\left(\mathrm{D}_{1}, \mathrm{D}_{2}\right)$, les abeilles seront happées régulièrement les jours suivants. Si les piqûres "douloureuses " surviennent, un potentiel inhibitif s'édifie, il tend à s'opposer à l'autre potentiel, à le diminuer et à l'empêcher de déclencher le comportement alimentaire. Son intensité augmente en fonction du nombre de piqûres, de leur fréquence et de leur effet douloureux.

Si l'inhibition est suffisamment forte, la punition prédomine, le Crapaud a appris à ne plus poursuivre l'Abeille-proie, il fuit la ruche.

On aboutit à une situation d'évitement. A ce moment-là un autre comportement s'amorce ; en évitant l'A beille, le Crapaud évite la punition et finalement celle-ci est supprimée. Comme le Crapaud n'est plus piqué, il n'y a plus de renforcement de l'apprentissage, la réaction d'évitement se dissipe ; au bout d'un certain temps, on en arrive à l'extinction de la punition.

Le Crapaud revient alors chasser 1'Abeille. S'il n'est pas piqué trop douloureusement, une habitude à l'Abeille-proie s'établit ou se rétablit. La récompense l'emporte 
sur la sanction mais le plus souvent, la consommation d'abeilles n'atteint pas celle des premiers jours. Fn avalant de nouveau les abeilles, les risques de piqûres augmentent, les punitions peuvent de nouveau s'accumuler, provoquant un nouvel apprentissage d'évitement. Chez beaucoup, on assiste alors à une certaine résistance à l'extinction de la récompense ; le Crapaud persiste à chasser l'Abeille, il ne l'évite pas complètement.

Puisqu'il y a alternativement extinction de la récompense et extinction de la punition, renforcement de la sanction et résistance (due à la faim) à l'extinction de la récompense, un refus systématique de la proie s'instaure difficilement, dure peu de temps, tandis qu'une adaptation positive toujours sujette à interruption peut s'établir.

L'Abeille est donc pour le Crapaud une proie ou un " danget ". Il y a conflit entre récompense et sanction.

Des situations semblables provoquent habituellement des névroses expérimentales chez le Singe, le Chien, le Chat et le Rat albinos (travaux de MILLER), (CAIN. I959). Ce type de comportement ne se manifeste pas chez le Crapaud, mais on peut noter le guet prolongé des abeilles avant de quitter la ruche, sans qu'il y ait eu tentative de happer. Cette " observation " est plus longue que dans les cas habituels de préhension, et elle n'a pas le caractère de "tension" si typique du temps d'arrêt.

Il arrive aussi que Bufo bufo se détourne d'une abeille après l'avoir regardée longuement. Au même moment il fait avec une de ses pattes antérieures un "geste latéral de frottement "; celui-ci n'est jamais effectué lorsque le Crapaud avale une Abeille mais lorsqu'une proie est rejetée parce qu'elle est trop amère ou trop grande et quand une brindille ou de la terre reste collée à la langue ou à la bouche. La tension accumulée se décharge donc par ce mouvement qui se déroule à vide. J'ai appelé "activité de compensation" (LESCURE, I965, I966) ce type de réaction de déplacement, où l'animal exécute un autre mouvement du même comportement et non un mouvement d'un autre comportement inné comme le picorage des Coqs pendant un combat ('TINBERGEN, I953).

Lorsque l'inhibition due aux piqûres douloureuses n'est pas suffisante pour l'emporter sur la force d'attraction de la proie, le Crapaud continue à chasser l'Abeille Sauf quelques cas, une piqûre douloureuse ne suffit pas, dans mes expériences, à déclencher la réaction d'évitement. La sanction semble moins efficace que la récompense, mais il est certain que, si les punitions s'accumulent, les captures diminuent Une meilleure preuve en est fournie par la comparaison de deux essais effectués avec les mêmes crapauds (tabl. 6, Série $A, D_{1}, D_{2}$ ). Dans l'un des deux essais, la ruche fut plus agressive et les piqûres douloureuses plus fréquentes, le nombre d'abeilles avalées fut moins grand.

A cause de la grande diversité des réactions des Crapauds aux piqûres d'Abeilles et de l'effet douloureux de ces piqûres, il n'est pas possible d'établir des courbes de la vitesse d'apprentissage d'évitement ou de la durée d'extinction de la punition en fonction de la fréquence des piqûres douloureuses. Une piqûre très douloureuse peut déclencher un refus de plusieurs jours, mais d'autre part, une piqûre douloureuse qui survient à la cinquième ou sixième abeille avalée n'a pas le même effet sur l'apprentissage à la punition que si elle était survenue à la première abeille. Chez certains individus $\left(A_{1}, C_{1}, E_{4}\right)$, la réaction d'évitement ne s'établit pas malgré de nombreuses piqûres douloureuses. 
Comme mes expériences s'effectuaient sur 9 jours, j'ai pu constater ce phénomène d'extinction de la punition et de la reprise de la capture des abeilles, même chez les individus qui avaient appris à fuir la ruche après une ou deux piqûres. Si l'on considère, en suivant les expériences de BuYTENDIJK (I9I8), SCHNEIDER (I954) et EIBL-EIBESFELDT (I95I), que deux ou trois jours suffisent pour fixer un dressage chez le Crapaud et que la réaction d'évitement ne se maintient généralement pas plus de 3 ou 4 jours, il faudrait admettre qu'un crapaud recommence un apprentissage nouveau le septième ou le huitième jour, après l'extinction d'une punition. Ma courbe de consommation d'abeilles parait corroborer une telle affirmation et correspond d'ailleurs au refus temporaire d'un certain nombre de mes crapauds. Ceux qui ont appris à éviter les Abeilles ne seraient donc pas ceux qui n’ont pas avalé d'abeilles le dernier jour de l'expérience, mais ceux qui, à la fin ou au cours de l'expérience, ont refusé les abeilles pendant 3 ou 4 jours consécutifs. C'est ainsi que Io de mes crapauds ont évité les abeilles pendant 4 jours ou plus et 3 pendant 3 jours.

TABLEAU 6

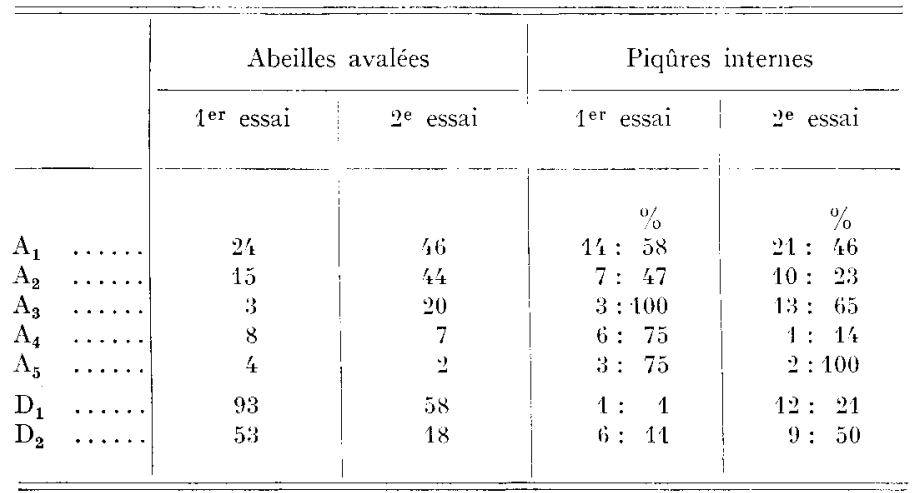

Ce refus de 3 ou 4 jours est la preuve que le dressage négatif s'est dûment établi ; on ne peut pas dire que les Crapauds ont appris à éviter les Abeilles, si on ne l'a pas constaté. Même si tous les crapauds expérimentés par CoTTi ont refusé les abeilles le septième jour, ils n'ont pas forcément appris à les éviter entièrement. Il suffit de prolonger l'expérience pour se rendre compte qu'ils recommencent à les guetter et à les avaler.

De toutes façons, 27 crapauds de CoTT sur 33 ne mangeaient plus d'abeilles les 3 derniers jours, alors qu'il y en a eu seulement 7 dans mes expériences. I,'écart entre nos résultats est tout aussi significatif si l'on compare les tracés de nos graphiques, c'est-à-dire ceux de ses figures I et 2 avec ceux de mes figures 3 et 4 .

Pourquoi une telle différence? Si mes expériences sont beaucoup moins négatives, c'est en grande partie parce que j'ai provoqué une diminution de la fréquence des piqûres douloureuses en changeant de conditions d'expériences. Le Crapaud se trouvait du même coup dans des conditions plus conformes à sa vie habituelle. Pendant les expériences de CớT'T les punitions se sont accumulées. On peut même se demander si en refusant de guetter les abeilles et en s'écartant de la ruche, les crapauds n'appre- 
naient pas à éviter une ruche bourdonnante, les vols d'abeilles, les piquires externes en même temps qu'une proie dangereuse qui provoque une piqûre douloureuse quand elle est avalée.

On a alors une double punition, l'une provenant de la ruche et de son agressivité, l'autre de 1'Abeille-proie. La première a été très importante dans les expériences de CoTT, les vols des abeilles ont gêné les crapauds et surtout 4 I piqûres externes ont été constatées. Leur effet est généralement radical : le lendemain la plupart des crapauds piqués ne mangent plus d'abeilles. Je l'ai remarqué moi-même au cours du $3^{\text {e }}$ essai de la série $A$ et avec $\mathrm{E}_{1}$; les crapauds qui avaient été piqués de cette façon quittaient la ruche aussitôt qu'ils étaient replacés devant.

Pourquoi les piqûres externes sont-elles plus efficaces que les internes? Est-ce parce que la douleur locale dans la peau et les muscles des pattes est plus forte que dans l'épithélium buccal? N'est-ce pas plutôt parce que la punition est isolée du contexte des stimuli alimentaires? Une abeille qui bourdonne vole autour d'un crapaud, le "houspille ", grimpe sur son dos et le pique, n'a pas signification de proie, tandis qu'une abeille qui pique dans le gosier pendant la déglutition est une proie. Il est donc normal que l'association ruche-Abeille-piqûre-externe soit plus forte et plus durable que l'association ruche-Abeille-proie-(bonne au goût)-piqûure-interne.

Des essais réalisés lorsqu'il y a peu de chances de piqûres externes, c'est-à-dire le soir, par un temps non pluvieux, devant une ruche assez calme (séries $\mathrm{D}, \mathrm{E}, \mathrm{F}$, $\mathrm{A}^{\prime \prime}=25$ Bufo) montrent que très peu de crapauds ( 4 sur 25 ) évitent systématiquement les abeilles et la ruche.

Si finalement, à cause des piqûres internes, un refus temporaire des Abeillesdevant-la-ruche peut s'établir assez facilement, je pense qu'aucun Crapaud n'apprend à les refuser totalement à cause de sa faible mémoire et de l'ambivalence de la proie. Celle-ci peut avoir soit la valeur innée de récompense alimentaire, soit la valeur acquise d'une punition qui souvent n'est pas assez forte pout déclencher un comportement de fuite durable.

\section{Adaptation du Crapaud d̀ l'A beille : infuence de la taille du prédateur}

Vis-à-vis de l'Abeille, Bufo bufo modifie son comportement prédateur. Peut-on discerner un critère qui permettrait de dire si tel crapaud a plus de chance de s'adapter aux Abeilles que tel autre? A la suite de mes expériences, je peux affirmer que cette adaptation est généralement fonction de la taille et du poids du crapaud.

En effet, le nombre d'abeilles avalées est fonction du nombre de piqûres internes "douloureuses".

Or, le nombre des piqûres dépend de deux facteurs, l'agressivité des abeilles et surtout " l'habileté " du crapaud. Les crapauds les plus " habiles » et les plus actifs, qui en même temps craignent moins le voisinage de l'homme, sont les grosses femelles (Io à I $2 \mathrm{~cm}$, plus de Ioo g). L'apprentissage et la vigueur musculaire ont augmenté la précision, la sûreté et la vitesse de leur coup de langue. Nous avons là un exemple typique de l'enchevêtrement de la maturation du mécanisme de visée et du happer (comportement inné) et de l'acquisition d'une plus grande habileté par les expériences individuelles (LEScuRE, I965).

La précipitation excessive chez les plus jeunes à s'élancer vers une proie, est plus 
rare et moins forte chez les grosses femelles. Elles attendront qu'une proie soit bien isolée des autres pour happer; certaines ont même des techniques de chasse très adroites et très effiaces.

D'autre part, la force du coup de langue qui assomme tout de suite l'Abeille, la vitesse de déglutition de la proie, sont autant de facteurs du comportement inné qui se sont perfectionnés avec l'âge.

Il résulte de tout ceci que les plus gros crapauds se font moins piquer et que, parfois, les dards des abeilles s'accrochent moins solidement dans leur gosier. Comme, en outre, ils sont moins sensibles à l'action de la piqûre et au venin lui-même, (cf. expériences p. 9r), l'Abeille n'est plus une proie très dangereuse pour eux; ils sont même susceptibles de s'y accoutumer.

Les résultats de mes expériences confirment cette possibilité d'adaptation des gros crapauds à l'Abeille. Les jeunes crapauds, au contraire, ne peuvent pas aboutir à une telle adaptation. A la figure 5, j'ai établi la courbe relative aux Io crapauds mesurant plus de Io $\mathrm{cm}$ et pesant plus de $100 \mathrm{~g}$ et celle relative aux animaux pesant moins de $80 \mathrm{~g}$ et mesurant moins de $9 \mathrm{~cm}$. Alors que pour ces derniers, le total du premier jour voisine avec les résultats des gros crapauds, ceux des jours suivants sont insignifiants. Par contre, les ro plus gros crapauds accusent un maximum s'étalant sur 3 jours, avec un sommet le deuxième jour, une baisse les quatrième, cinquième et sixième jours et une remontée ensuite.

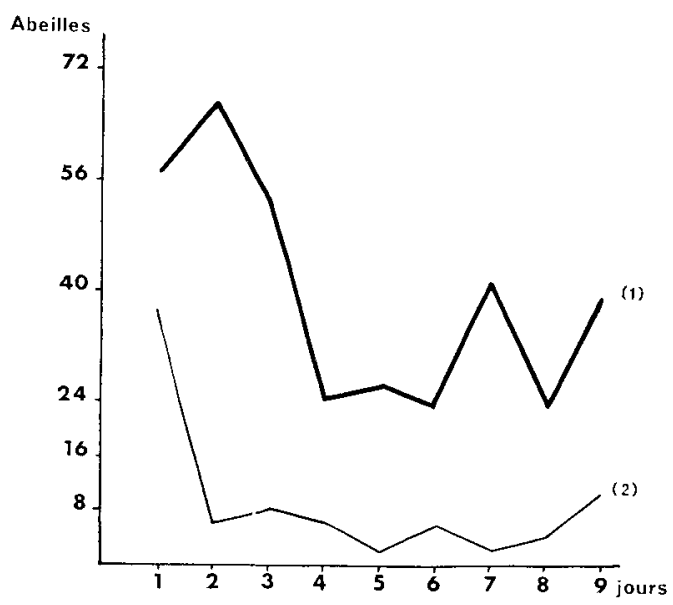

FIG. 5. - Nombre d'abeilles avalées chaque jour soit par les ro plus gros crapauds $(+$ de $10 \mathrm{~cm},+$ de $100 \mathrm{~g})$, Courbe (1) soit par les ro plus petits crapauds (- de $9 \mathrm{~cm}$, - de $80 \mathrm{~g})$, Courbe (2)

Ces deux courbes sont très significatives si on les compare avec la courbe de la figure I de CoT'T. La courbe (2), excepté son extrémité, a la même signification que celle de CoTT : une chute brusque de la consommation d'abeilles au bout de quelques jours, mais celle-ci n'en aboutira pas pour autant à un refus total. La courbe (I) n'a aucun point de comparaison avec celle de CoTT. Celui-ci indique que ses sujets d'expérience étaient tous adultes et il semble d'après les exemples cités que ses crapauds mesuraient de 70 à $80 \mathrm{~mm}$. Les plus grands mâles que Smitrr (I95I) a trouvés en 
Angleterre atteignent $70 \mathrm{~mm}$, et les femelles $87 \mathrm{~mm}$, et il ajoute que "dans le sud de 1'Europe, le Crapaud est beaucoup plus grand que dans les Iles Britanniques".

Je pense que c'est dans la différence de taille des crapauds utilisés que réside une des principales raisons de la divergence de nos résultats. C'est en expérimentant avec de grosses femelles de $B$ ufo bufo qu'on peut dégager des résultats nettement positifs. Ce ne sera pas leur grande voracité mais leur " habileté » qui expliquera la différence d'avec les petits crapauds adultes. La lecture de la figure 6 indiquant, par jour, le nombre des Io plus gros et des so plus petits crapauds mangeant des abeilles, révèle bien cette différence d'attitude.

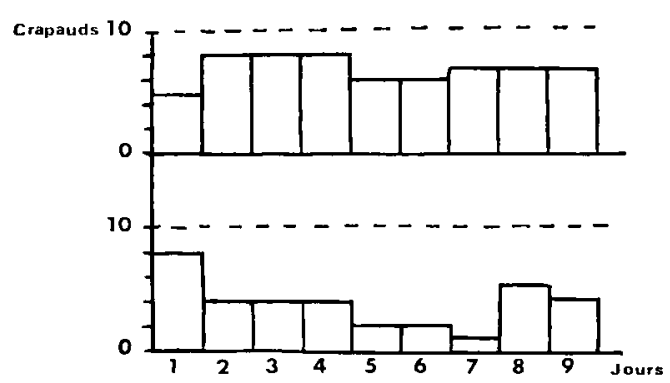

FIG. 6. - Nombre de crapauds avalanl des abeilles chaque jour

a) En haut, parmi les ro plus gros crapauds

b) En bas, parmi les to plus petits crapauds

L'adaptation à une proie dangereuse comme 1'Abeille est donc fonction du poids et de la taille de Bufo bufo. De grosses femelles de I i cm et pesant dans les $200 \mathrm{~g}$ comme $\mathrm{D}_{1}, \mathrm{D}_{2}$ et $\mathrm{C}_{1}$ qui était borgne, en sont des exemples frappants. Les petits Crapauds adultes $(6$ à $8 \mathrm{~cm}$, moins de $70 \mathrm{~g})$ n'arrivent pas à s'adapter à une telle proie, même s'ils ne la refusent pas totalement. Leur inadaptation peut s'expliquer de diverses façons. Généralement, ils ne s'approchent pas très près des orifices de la ruche et le comportement de fuite l'emporte facilement sur les réactions alimentaires. Leur coup de langue n'est pas assez fort pour bien assommer l'Abeille, et celle-ci pique presque à chaque coup. Même lorsqu'ils chassent 1'Abeille, je n'ai jamais observé en eux l'habilité des grosses femelles; mais je pense que c'est moins par impossibilité psychique que par la faiblesse de leur coup de langue que les petits Bufo adultes ne peuvent pas s'habituer à une telle proie. Quant aux crapauds moyens $(\delta$ à ro $\mathrm{cm}$ ), leur comportement de fuite n'est pas aussi fort. Le premier jour, ils paraissent même plus " excités " que les gros crapauds ; pour la même durée de jeûne, leur faim semble plus grande, le "mobile" de leur réaction (drive) plus fort. Cet "énervement " provoque des réactions de happer trop rapides. Les abeilles piquent presque à chaque fois.

Le tableau 7 donnant le nombre d'abeilles avalées chaque jour par les crapauds et où ceux-ci sont classés par ordre de taille et de poids, nous indique bien l'importance de ces paramètres. Pourquoi alors ne pas conclure en affirmant que cette adaptation est finalement fonction de l'âge? Je ne peux par tirer une telle conclusion, car la différence de grandeur entre les deux sexes est prononcée chez Bufo bufo. REy (I944) a relevé les mensurations sur une population de Bufo bufo dans le Jura, les I72 ๙ $^{x}$ allaient de 46 à $82 \mathrm{~mm}$ et les $84 q$ de 65 à Io $2 \mathrm{~mm}$. Dans le midi de la France, les $q$ peuvent être encore plus grandes. Si l'on est certain qu'un crapaud dépassant Io $\mathrm{cm}$ 
est une femelle âgée, on ne sait pas quelle est exactement la limite supérieure de la taille des mâles. C'est pourquoi je préfère garder comme critères, la taille et le poids du Crapaud, grandeurs mesurables.

TABLEAU 7

Nombre d'abeilles avalées chaque jour par chaque crapaud

Les Bufo bufo sont classés par ordre de taille décroissante

\begin{tabular}{|c|c|c|c|c|c|c|c|c|c|c|c|}
\hline & Crapauds & $1^{\mathrm{er}}$ jour & 2 jour & $3^{e}$ jour & $4^{\mathrm{e}}$ jour & $5^{\mathrm{e}}$ jour & $6^{\mathrm{e}}$ jour & $7^{\mathrm{e}}$ jour & ge jour & ge jour & Total \\
\hline & $D_{1}$ & 32 & 3 & 8 & 0 & 15 & t & 8 & 9 & 16 & 95 \\
\hline & $\mathrm{C}_{1}$ & 0 & 0 & 10 & 6 & 1 & 3 & 7 & 1 & s & 36 \\
\hline & $\mathrm{D}_{2}$ & 12 & 7 & 8 & 7 & 4 & 7 & 4 & 2 & 2 & 53 \\
\hline & $D_{3}$ & 0 & 0 & 8 & 0 & 0 & 0 & 0 & 0 & 0 & 8 \\
\hline & $D_{5}$ & 5 & 9 & 2 & 4 & 0 & 0 & 0 & 1 & 3 & $y^{\prime} x^{\prime}$ \\
\hline \multirow[t]{6}{*}{$11 \mathrm{~cm}$} & & & & & & & & & & & \\
\hline & $D_{4}$ & 0 & 5 & 1 & 1 & 0 & 0 & 0 & 0 & 0 & 7 \\
\hline & $\mathrm{D}_{8}$ & 5 & 22 & 0 & 1 & 1 & 1 & 4 & 2 & 0 & 36 \\
\hline & $D_{7}$ & 0 & 6 & 2 & 1 & 3 & 7 & 7 & 1 & 't & 31 \\
\hline & $\mathrm{D}_{6}$ & 3 & 8 & 0 & 1 & 0 & 1 & 4 & 7 & $i$ & 29 \\
\hline & $13_{1}$ & 0 & 7 & 13 & 3 & 2 & 0 & 7 & 0 & 1 & 33 \\
\hline \multirow{5}{*}{$10 \mathrm{~cm}$} & & & & & & & & & & & \\
\hline & $C_{2}$ & 0 & 0 & 0 & 0 & 6 & 5 & 9 & 0 & 2 & 29 \\
\hline & $D_{9}$ & 1 & 3 & 10 & 0 & 0 & 0 & 0 & 0 & 3 & 17 \\
\hline & $\mathrm{D}_{10}$ & 1 & 0 & 4 & 1 & 2 & 0 & 1 & 0 & 1 & 10 \\
\hline & $A_{1}$ & 1 & 2 & 7 & $\prime^{\prime}$ & 3 & 2 & 0 & 1 & 主 & $2^{\prime} t$ \\
\hline $100 \mathrm{~g}$ & $\mathrm{E}_{1}$ & 9 & 0 & 0 & 0 & 0 & 0 & 0 & 1 & 2 & 12 \\
\hline \multirow[t]{5}{*}{$9 \mathrm{~cm}$} & & & & & & & & & & & \\
\hline & $A_{2}$ & 0 & / & 3 & 7 & 0 & 1 & 0 & 0 & 0 & 15 \\
\hline & $F_{1}$ & 3 & 2 & 3 & 0 & 2 & 0 & 0 & 2 & 1 & 13 \\
\hline & $\mathrm{E}_{2}$ & 7 & 0 & 0 & 0 & 0 & 0 & ) & 0 & 0 & 7 \\
\hline & $F_{2}$ & 3 & 2 & 1 & 0 & 1 & 1 & 3 & 3 & t & 18 \\
\hline \multirow[t]{2}{*}{$90 \mathrm{~g}$} & & & & & & & & & & & \\
\hline & $F_{3}$ & 0 & 1 & 0 & 1 & 1 & 0 & 0 & 1 & 0 & 's \\
\hline \multirow[t]{5}{*}{$80 \mathrm{~g}$} & & & & & & & & & & & \\
\hline & $\mathrm{E}_{3}$ & 13 & 0 & 1 & 2 & 0 & 0 & 0 & 1 & 0 & 17 \\
\hline & $\mathrm{E}_{4}$ & 13 & 2 & 1 & 2 & 1 & 3 & 2 & 1 & 2 & 27 \\
\hline & $\mathrm{F}_{4}$ & 1. & 0 & 0 & 1 & 1 & 2 & 0 & 0 & 1 & 6 \\
\hline & $\mathrm{E}_{5}$ & t & 0 & 0 & 0 & 0 & 0 & 0 & 1 & 2 & 7 \\
\hline \multirow[t]{3}{*}{$8 \mathrm{~cm}$} & & & & & & & & & & & \\
\hline & $A_{3}$ & 1 & 1 & 0 & 1 & 0 & 0 & 0 & 0 & 0 & $: 3$ \\
\hline & $\mathrm{A}_{4}$ & 1 & 2 & 5 & 0 & 0 & 0 & 0 & 0 & 0 & 8 \\
\hline \multirow[t]{5}{*}{$7 \mathrm{~cm}$} & & & & & & & & & & & \\
\hline & $\mathrm{C}_{4}$ & 0 & 0 & 0 & 0 & 0 & 0 & 0 & 1 & 0 & 1 \\
\hline & $\mathrm{B}_{2}$ & 2 & 0 & 0 & 0 & 0 & 0 & 0 & 1 & 0 & 3 \\
\hline & $\mathrm{C}_{3}$ & 0 & 0 & 0 & 0 & 0 & 0 & 0 & 0 & 5 & 5 \\
\hline & $A_{5}$ & 2 & 1 & 1 & 0 & 0 & 0 & 0 & 0 & 0 & 4 \\
\hline & & 119 & 87 & 88 & 43 & 13 & 37 & 56 & 36 & 66 & 575 \\
\hline
\end{tabular}

Si on utilise ces paramètres (la taille et le poids) avec d'autres espèces de Bufonidés, on peut faire concorder les conclusions de mes expériences avec les observations effectuées sur d'autres espèces et faire en même temps justice d'apparentes contradictions. La taille moyenne des Crapauds d'Angleterre ( 60 à $80 \mathrm{~mm}$ ) explique en 
grande partie les résultats de Cor'r. Par contre, les adaptations positives qui ont été observées concernent de plus gros crapauds. Les Crapauds de Californie récoltés par ECKERT (I934) peuvent atteindre $13,5 \mathrm{~cm}$. Les faits rapportés à CHAUVin par les apiculteurs d'Algérie et d'Amérique du Sud, concerneraient soit Bufo bufo spinosus $(\mathrm{I} 5 \mathrm{~cm})$ soit Bufo marinus ( $18 \mathrm{~cm})$, le plus gros des Crapauds. A l'île Martinique, Bufo marinus est un peu plus petit $(15 \mathrm{~cm})$, mais il est tout aussi néfaste aux Abeilles.

\section{Importance du tacteur individuel dans le comportement d'adaptation aux Abcilles}

Èn lisant le tableau 7 , on s'aperçoit que dans un groupe de crapauds de même taille les résultats sont très divers. Parfois, ces crapauds sont de la même série et ont donc subi les mêmes conditions d'expérience (agressivité de la ruche, temps). On constate, par exemple, que tous les gros crapauds ne s'habituent pas à chasser les abeilles devant une ruche. Certains ont été réfractaires à une telle adaptation. Pourquoi? Certes, le cas de $\mathrm{D}_{3}$ est typique en son genre : suivant ses réactions, la manipulation de l'expérimentateur semble bien être la cause de sa fuite instantanée devant la ruche. Mais pourquoi $A_{1}$ s'est-il mieux adapté que $D_{10}$ et, dans la même série, $\mathrm{I}_{2}$ que $\mathrm{D}_{4}$ et $\mathrm{I}_{5}$ ?

L'explication en est difficile. Une piqûre plus douloureuse au $4^{\mathrm{e}}$ jour a pu être le motif du refus de $\mathrm{I}_{4}$ durant 3 jours, mais $\mathrm{D}_{2}$ s'est pourtant fait piquer le même jour et il a continué à capturer les abeilles; $\mathrm{D}_{2}$ était peut-être plus résistant ou la piqûre moins douloureuse. Quant à $\mathrm{D}_{4}$, des signes certains de douleurs dus à des piqûres internes sont apparus après l'absorption d'une abeille les troisième et quatrième jour ; aussitôt après, il fuyait la planche d'envol. A l'opposé, malgré ces mêmes réactions de malaise, des crapauds plus petits conme $\mathrm{E}_{1}$ se sont acharnés chaque jour à guetter et à avaler des abeilles. Il est certain que la diversité des résultats, quant aux nombres d'abeilles avalées, peut s'expliquer par l'ampleur des effets douloureux des piqûres, mais cela ne suffit pas. $\mathrm{L}_{4}$ g grande variété des réactions des crapauds en face des abeilles nous oblige à constater en même temps l'importance du facteur individuel dans un tel comportement.

Parmi les crapauds de taille moyenne ( 8 à $10 \mathrm{~cm}, 80$ à $100 \mathrm{~g}$ ), l'importance du facteur individuel est encore plus évident dans les résultats que pour les gros et les petits spécimens. Alors que les grosses femelles s'orientent généralement vers l'adaptation et les petits Bufo vers le refus, ceux de taille moyenne sont à la limite. Les individus qui s'adaptent réellement sont rares $\left(A_{1}, A_{2}, 2^{\mathrm{e}}\right.$ essai). Malgré les piqûres des jours précédents, certains chassent les abeilles avec la même ardeur à chaque nouvelle séance ; une seule piqûre ou une piqûre plus douloureuse à la deuxième, troisième ou quatrième abeille avalée les chassera $\left(\mathrm{E}_{\mathfrak{l}}, \mathrm{F}_{2} ; \mathrm{A}_{1}, \mathrm{I}^{\mathrm{er}}\right.$ essai). D'autres n'ont pas un comportement bien définissable $\left(\mathrm{D}_{10}, \mathrm{~F}_{1}\right)$.

Dans ce facteur individuel, il faut inclure d'abord ce coefficient individuel d'activité ou de vivacité que j'ai décrit comme une variable innée du comportement prédateur (LESCURE, I965). Bien qu'ils fussent souvent piqués, quelques individus plus actifs continuèrent tous les jours à chasser la dangereuse proie $\left(\mathrm{E}_{1}, \mathrm{~A}_{1}\right)$. $\mathrm{C}_{1}$ qui était borgne, se faisait très souvent piquer parce qu'il attrapait les abeilles au vol, ou par un coup de langue trop latéral, ou bien lorsqu'elles lui passaient sur 
la bouche. Cela ne l'empêchait pas de recommencer à chasser chaque jour et de se retourner vivement lorsqu'une abeille traversait son champ visuel.

L'importance du facteur individuel se manifeste encore plus dans " l'habileté » du Crapaud. J'ai déjà dit comment l'inné et l'acquis se mêlent sous ce mot. La part du comportement acquis se révèle souvent dans les méthodes de chasse du Crapaud devant la ruche. Dès le premier jour, un crapaud comme $D_{1}$ avait une technique très efficace. Placé sur la plate-forme, il la traversait pour se placer légèrement à gauche devant un orifice de sortie, regardait les abeilles à l'intérieur et lorsque l'une d'elles débouchait, le coup de langue était déjà parti. Dans un deuxième essai effectué I 5 jours plus tard, $D_{1}$ réoccupa la même position. Sans précipitation, $D_{2}$ et $A_{1}$ se mettaient plutôt de côté sur la planche d'envol. Ils guettaient presque toujours le même orifice de sortie ou la même surface de la planche, happaient les abeilles isolées qui arrivaient en face d'eux et laissaient passer les abeilles qui arrivaient latéralement ou par derrière. $\mathrm{A}_{3}$, lors de son $2^{\mathrm{e}}$ essai, adopta une place à l'extrémité nord-gauche de la plate-forme. Pendant ro jours, qu'il soit mis du côté droit ou du côté gauche, il traversait la planche d'envol pour rejoindre sa place habituelle et y attendre les abeilles.

Ia persistance des techniques de chasse de plusieurs crapauds indique aussi qu'un autre élément individuel, la mémoire (capacité et méthodes d'enregistrement) joue un grand rôle dans le comportement de chaque Bufo bufo devant la ruche.

Il faut tout d'abord noter que le Crapaud apprend à éviter une proie ou un danger, par quelques repères localisés dans le champ perceptif. Ces repères, qui deviennent des stimulis de la réaction d'évitement, sont le plus souvent un ou deux caractères de la proie et non l'ensemble de la proie. Ils sont rarement retenus d'une façon totalement isolée, mais enregistrés dans un contexte donné : décor, local, forme de l'attrape (EIBI-EIBESFEI,DT, I95I). Le signal de la sanction à fuir sera donc lié à une association, caractère de la proie-décor par exemple: il suffira qu'un élément de l'association manque pour que l'autre élément ne soit plus un signal de sanction. LF Ny (I96I) ne pense pas autre chose quand il dit que dans une situation d'évitement, l'extinction de la réaction a lieu lorsque la situation elle-même change. D'autres fois, le repère de conditionnement n'est pas même pris sur la proie mais dans d'autres parties du champ visuel.

Avec de pareilles méthodes d'apprentissage, le signal du refus n'est pas nécessairement attaché à l'A beille ou à l'A beille seule. Un soir, après la dernière expérience du troisième essai de la série $A\left(A^{\prime \prime \prime}\right)$, des abeilles présentées dans le vivarium furent avalées aussitôt, alors que ro minutes plus tôt elles étaient évitées devant la ruche. J'ai pu renouveler deux fois cette observation. Le caractère danger était donc associé à la ruche et non à l'Abeille, ou inclus dans une association plus grande Abeille-ruche.

Il est toutefois certain que dans un cadre invariable d'expérience, le Crapaud peut aussi reconnaître 1'Abeille parmi d'autres proies par une discrimination fondée sur sa forme, ses couleurs ou son mouvement. Lors du même essai de la série A, des Fourmis qui couraient sur le ciment devant les ruches étaient poursuivies et happées alors qu'à $50 \mathrm{~cm}$ de là, les abeilles n'étaient pas chassées. Pour ces crapauds-là, le caractère danger était associé à l'Abeille elle-même.

$\mathrm{Si}$ ces associations mémorielles diffèrent pour chaque individu, leur persistance varie aussi d'un crapaud à l'autre. La réaction d'évitement s'éteint plus ou moins vite et surtout elle est renouvelée ou renforcée plus ou moins rapidement et plus ou 
moins longuement par quelques expériences " négatives ". EIBI-EIBESFEL,DT l'a constaté alors qu'il travaillait avec des attrapes neutres au goût ; il n'avait donc pas à tenir compte des variations d'effets douloureux comme dans mes expériences. II est très exact, comme CoTT (I936) l'avait affirmé, que la capacité d'apprentissage de Bufo bufo varie beaucoup d'un sujet à l'autre.

Au sujet des variations individuelles de la mémoire de Bufo bufo, les exemples les plus frappants sont les résurgences, d'un essai à l'autre, de ces méthodes de chasse que j'ai évoquées plus haut. Lors d'un deuxième essai, certains de mes gros

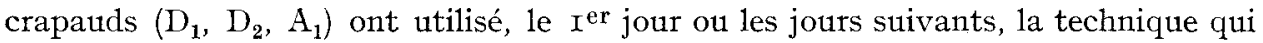
leur avait réussi au premier essai. Ils allèrent occuper la même place et guettèrent de nouveau devant les orifices de sortie.

Il y aurait donc une certaine permanence de l'association ruche-Abeille-méthode de chasse, de la même façon que les Crapauds s'habituent à happer les Vers de terre par les mâchoires et non par la langue. A ces exemples, il faudrait ajouter les cas de retour à la ruche observés dans la nature, notamment celui examiné par le Dr MoREAUX avec sa technique bien particulière (cf. p. 86). Il semble donc que le Crapaud varie sa technique de prédateur suivant les situations. A cause du petit nombre de repères qu'il utilise pour fixer son souvenir, je pense qu'il peut apprendre à discerner l'association ruche-Abeille-méthodes-de-chasse d'une association Abeille-piqûre. I1 se pourrait même que la mémorisation soit meilleure dans ce cas que dans celui de la piqûre : il s'agit, en effet, d'un souvenir améliorant la récompense ; tandis que dans l'autre cas c'est une réaction d'évitement à extinction plus rapide. $\mathrm{D}_{1}$ et $\mathrm{D}_{2}$ avalèrent moins d'Abeilles à leur deuxième essai, car ils furent davantage piqués (cf. tab1. 6) mais ils conservèrent leur méthode de chasse. La permanence de ces associations d'une méthode de chasse avec une proie ou une situation est d'ailleurs un des principaux facteurs de l'expérience individuelle, de l' " habileté " du Crapaud.

En revanche, je suis obligé de mettre en doute l'interprétation de CoT'T pour une expérience qui aboutit pourtant au même type de conclusions. Avec I 8 crapauds, CotT recommença un deuxième essai I 5 jours après le premier. La consommation d'abeilles fut nettement plus faible que la première fois. Il en conclut alors que la leçon du refus a été apprise plus rapidement parce que la leçon précédente avait été partiellement ou entièrement retenue pendant la quinzaine de jours qui séparait les deux essais.

S'agirait-il d'une sorte de "récupération "d'un apprentissage comme l'a décrit LE Ny (I96I) dans son étude sur le conditionnement? C'est possible, mais la preuve apportée par CoT'T n'est pas décisive. En effet, les trois essais que j'ai effectués avec la série $A$, le deuxième essai avec $D_{1}$ et $D_{2}$, des essais partiellement répétés avec la série F, m’obligent à dire qu'à chaque nouvel essai réalisé après un intervalle de I5 jours, les crapauds apprenaient à accepter ou à refuser les abeilles suivant la fréquence des piqûres douloureuses, les conditions d'expérience et le temps de jê̂ne supporté. Le cas de la série A (cf. tabl. 5 p. $9^{8}$ ) en est un exemple typique Quatre crapauds sur 5 avaient appris à éviter les abeilles au premier essai; au second, les conditions étant meilleures, ils apprirent à les avaler tandis qu'au troisième, ils recommencèrent à les éviter, les ruches étant plus agressives.

Dans son attitude en face de 1'Abeille, il semble donc que le rôle du coefficient individuel de Bufo bufo est considérable : degré d'activité, d' " habileté " au happer, techniques de chasse et enfin méthodes et possibilités de sa mémoire. 


\section{V. - CONCLUSION}

De ces expériences sur le comportement prédateur de Bufo bufo devant une ruche, on peut tirer les conclusions suivantes:

I. Il n'y a pas de refus instinctif de l'Abeille de la part de Bufo bufo.

2. Peu de Crapauds apprennent à éviter systématiquement les Abeilles et aucun ne les refuse totalement.

3. Dans des conditions normales de chasse (le soir), le Crapaud adapte son comportement de prédateur à l'égard de l'Abeille devant la ruche.

4. Le sens de cette adaptation est fonction de la taille et du poids du Crapaud.

a) Les petits crapauds (moins de $8 \mathrm{~cm}$ et de $70 \mathrm{~g}$ ) avalent peu d'abeilles et apprennent rapidement à les éviter.

b) Les crapauds de taille moyenne $(8$ à $10 \mathrm{~cm})$ ont des comportements individuels très variables. Beaucoup d'entre eux se précipitent sur les abeilles le premier jour et les évitent ensuite périodiquement.

c) Parmi les grosses femelles (plus de io $\mathrm{cm}$, plus de roo g), beaucoup (8/10) s'adaptent à cette proie. Ceci correspond à des faits observés dans la nature.

5. Le nombre d'abeilles avalées et donc l'adaptation est fonction du nombre de piqûres douloureuses reçues. Le nombre de piqûres externes dépend principalement de l'agressivité de la ruche, tandis que le nombre de piqûres internes dépend à la fois de l'agressivité de chaque abeille et de 1' " habileté " des crapauds.

6. Le coefficient individuel de chaque crapaud joue un tôle important dans la modification de son comportement prédateur.

Il est donc certain que les Crapauds communs peuvent s'habituer à une proie dangereuse comme l'Abeille. Mes expériences viennent ainsi vérifier les affirmations des apiculteurs et répondre à la question que pose DotTrexs (r963) dans son livre sur les Batraciens et Reptiles de l'Europe. Si mes conclusions s'avèrent bien différentes de celles de COTT, c'est principalement dû̀ à deux modifications des données de l'expérience. J'ai opéré le soir et la nuit, heure de chasse du Crapaud et j'ai travaillé avec des individus adultes de toute taille.

Mes expériences fournissent en même temps un exemple d'adaptation, de dressage " positif " vis-à-vis d'une proie. Cependant parce que la capture de l'Abeille peut aussi bien entraîner une récompense qu'une punition (piqûre), si un dressage négatif n'est jamais définitif (Bufo oublie vite), un dressage positif peut aussi s'atténuer ou être interrompu.

L'étude du comportement de Bufo bufo devant une ruche confirme aussi le fait que ce Batracien est capable d'opérer une sélection parmi les proies qu'il rencontre (CoTT, I936, I940; LESCURE, I964, I965). Cependant la mémorisation des proies à éviter n'est jamais définitive, elle doit être renouvelée périodiquement. Le nouvel apprentissage est peut-être plus rapide (CoTT, I936) mais il dépend surtout de la force de la punition (caractère non comestible de la proie ou " douleur " de la piqûre) 


\section{REMERCIEMENTS}

Ce travail a été dirigé par M. le Pr Chauvin, ses encouragements et ses conseils m'ont constamment éclairé pendant ines recherches.

Plusieurs expériences ont été effectuées à la Station de Recherches sur l'Abeille et les Insectes sociaux de Bures-sur-Yvette ; l'aide procurée me fut très précieuse. Je remercie vivement M. le Directeur de la Station et ses collègues pour leur hospitalité bienveillante et amicale.

\section{SUMMARY}

\section{THE PREDATORY BEHAVIOUR OF “ BUFO BUFO » TOWARDS BEES}

Apis mellifica is a double-meaning prey for the Toad because she is at the same time a signal of recompense (good to taste) and a signal of punishment (the sting). So as to study the predatory behaviour of Bufo bufo before the hive, 30 adult Toads of varying sizes were used. The experiments were carried out in the evening at the time when the Toad hunts and when the hive is quieter. The Batrachians that had been fasting for several days were placed on the platform of a hive on 9 consecutive evenings. The consummation of Bees diminished very much on the 4 th, 5 th and 6 th days and then from the 7 th day it regained, without however arriving at the maximum of the first days.

A very clear and definite distinction has to be established between the small Toads (less than $9 \mathrm{~cm}$ and weighing $90 \mathrm{~g}$ ) and the large females (more than $10 \mathrm{~cm}$ and weighing $\mathrm{I} 00 \mathrm{~g}$ ). The first had themselves "painfully " stung by practically all the Bees that they swallowed; they learn quickly to avoid them, but from time to time they begin anew to catch them. The reaction of avoidance is blotted out by the lack of reinforcement, but it is renewed as soon as the little Toad catches a Bee and has been stung.

The large female Toads are more "capable " and can resist better the venom of the bees; the stings are less frequent because of the strength and precision of their tongue-stroke, they are also less "painful". These females can get used to a prey such as the Bee (a specimen of I $15 \mathrm{~mm}$ and weighing $200 \mathrm{~g}$ ate 32 bees in one hour and 95 in nine evenings). As a result of more painful stings, a reaction of avoidance can be established, but after its extinction a new adaptation may be constituted. More often than not the effect of these stings manifests itself by a momentary diminution in the consummation of Bees.

The great variety in the reactions of Toads (especially in the case of those of medium size) obliges us to establish, with regard to such varied behaviour, the importance of the individual factor (the coefficient of vivaciousness, capability and acquired behaviour).

From these experiments we may draw the following conclusions : r) There is no instinctive refusal of the Bee on the part of Bufo bufo. 2) Few Toads learn to avoid the Bees systematically, and no Toad refuses them entirely (the experiments of CoTT, $193^{6}$, are discussed). 3 ) In the normal hunting conditions (the evening), the Toad adapts his behaviour, with regard to the Bee, before the hive. The particular form of this adaptation is determined by the size and weight of the Toad. Only the large female Toads (more than Io $\mathrm{cm}$ or weighing more than $\mathrm{IOO} \mathrm{g}$ ) can really adapt themselves to this prey. This is confirmed by facts observed in nature. 4) The number of Bees swallowed is determined by the number of painful stings received; the number of stings depends on the aggressiveness of the Bees and on the dexterity of the Toad.

\section{RÉFÉRENCES BIBLIOGRAPHIQUES}

Alpinandery G., I946. Eurcyclopédie apicole. fasc. I1, ?. 374, Baillere. Paris. Bollenger G. A., 19ro. Les Batraciens, p. 231, Doin, Paris.

BRAGG A. X., I948. An observation showing biological necessity for size selection of food objects by young Toads. Proc. Okla. Acad. Sci., 28, 35-36.

Buttendjk F. J. J., rgio. L'instinct d'alimentation et l'expérience chez les Crapauds. Arch. Néerl.

Sci. exact. et natur. série, i I . C. 2, p. 2 I 7-228.

Buytendijk F. J. J., ig28. Psychologie des Animanx. 3 I 5 p., Payot, Paris.

Annales de l'Abeille. - Ig66. 
CaIllas A., 1948. Le Rucher de rapport. p. $186,3^{\mathrm{e}}$ édit, Orléans.

CAIN J., 1959. Le problème des névroses expérimentales. Desclées de Brouwer, Bruges.

Chacvin R., 1956. Vie et Mours des Insectes. p. 21 3, Payot, Paris.

Collin de Plancy V., i876. Recherches sur l'alimentation des Repliles et Batraciens de France. 31 p., Paris.

CotT II. B., 1936. The effectiveness of protective adaptations in the Hive-Bee illustrated by experiments on the feeding reactions, habit formation and memory of the common Toad. Proc. zool. Soc. London, in II33.

CotT H. B., 1940. Adaplive Coloration in Animals. 508 p., . Wethuen, Londres.

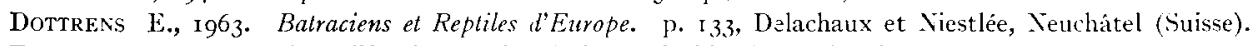

Eckert J. E., 1934. The California Toad in relation to the hive-hee. Copeia, 2, p. 92-3.

Eibl-Iinesfeldt I., I95I. Nahrungserwerb und Beuteschema der Erdkröte. Behaviour, I-35.

FA'T1O V., I872. Faune des Vertébrés de la Suisse. 3. Reptiles et Batraciens, p. 396, Georg, Genève et Bâle.

GIRARD M., I878. Calalogue raisonné des animaux utiles el muisibles de la France. 2 vol., Hachette, Paris.

GRANT Ch., 1948. Selection between armed and unarmed Arthropods as food by various animals. $S$. Ent. zool. Claremont., Cal 40, p. 66.

Héron-Royer., i879. De l'utilité des Batraciens Anoures. (Extr. Bull. Soc. zool. Fr., i878, p. 286-298), Paris.

La Fontaine A. de, i87o. Faune du Pays de Luxembourg: Reptiles., p. 37, Van Buck, Luxembourg.

LeComte J., 1961. Le comportement agressif des ouvrières d'. I pis mellifica L. Ann. Abeille, 4, (3). 165-270.

Le NY J. F., ig6r. Le Contilionnemenl. 172 P., P. U. F., Paris.

Lescure J., ig64. J'Alimentation du Crajaud commun, Bufo bufo. Vie et Milieu, 15, (3), 757-764.

Lescurf J., 1965. L'Alimentation et le C'omportement de prédation chez Bufo bufo. 164 p., Thèse Fac. Sci., Paris.

Lescure J., ig66. Le comportement alimentaire de Bufo bufo. Rôle des membres antérieurs daus la préhension des proies. La Terre et la Vie 7,3-76.

Matils M., I94I. L'exploitation ralionnelle des Abeilles. p. 94, P. U. F., Paris.

Phisalix C., I go4. Recherches sur le venin d'Abeilles. C. R. Soc. Biol, 139. p. 325.

Phisalix II., I922. Animalex venimeux el Venins. 2 vol. Masson, Paris.

REY P., 1946. Sur l'existence de races géoyraphiques chez Bufo mul garis Lavr. Bull. Soc. sool. Fr, 71, p. 190-3.

Rostand J., 1933. La vie des Crapauds. 220 p., $2^{\mathrm{e}}$ édition, 1947 ), Stock, Paris.

Schneider D., I954. Beitrag zu einer Analyse des Beute-und Fluchtverhaltens einheimischer Anuren Biol. Z., 78, p. $225^{-282 .}$

Surtr M., r95. The British Amphibians and Reptiles. 319 P.o, Collins, Loudon.

Tinbergen N., I953. L'Étude de l'instincl. 308 p., Payot, Paris.

W du Ilusée royal d'Hist. nat. de Belg., Bruxelles. 